\title{
Werner's Measure on Self-Avoiding Loops and Welding
}

Angel CHAVEZ and Doug PICKRELL

Mathematics Department, University of Arizona, Tucson, AZ 85721, USA

E-mail: achavez@math.arizona.edu,pickrell@math.arizona.edu

URL: http://math.arizona.edu/ achavez/, http://math.arizona.edu/ pickrell/

Received February 18, 2014, in final form July 31, 2014; Published online August 04, 2014

http://dx.doi.org/10.3842/SIGMA.2014.081

\begin{abstract}
Werner's conformally invariant family of measures on self-avoiding loops on Riemann surfaces is determined by a single measure $\mu_{0}$ on self-avoiding loops in $\mathbb{C} \backslash\{0\}$ which surround 0. Our first major objective is to show that the measure $\mu_{0}$ is infinitesimally invariant with respect to conformal vector fields (essentially the Virasoro algebra of conformal field theory). This makes essential use of classical variational formulas of Duren and Schiffer, which we recast in representation theoretic terms for efficient computation. We secondly show how these formulas can be used to calculate (in principle, and sometimes explicitly) quantities (such as moments for coefficients of univalent functions) associated to the conformal welding for a self-avoiding loop. This gives an alternate proof of the uniqueness of Werner's measure. We also attempt to use these variational formulas to derive a differential equation for the (Laplace transform of) the "diagonal distribution" for the conformal welding associated to a loop; this generalizes in a suggestive way to a deformation of Werner's measure conjectured to exist by Kontsevich and Suhov (a basic inspiration for this paper).
\end{abstract}

Key words: loop measures; conformal welding; conformal invariance; moments; Virasoro algebra

2010 Mathematics Subject Classification: 60D05; 60B15; 17B68; 30C99

\section{Introduction}

Given a topological space $S$, let $\operatorname{Comp}(S)$ denote the set of all compact subsets of $S$ with the Vietoris topology, and let

$$
\operatorname{Loop}(S):=\left\{\gamma \in \operatorname{Comp}(S): \gamma \text { is homeomorphic to } S^{1}\right\}
$$

with the induced topology (see Appendix A). Suppose that for each Riemann surface $S, \mu_{S}$ is a positive Borel measure on $\operatorname{Loop}(S)$. Following Werner, this family of measures is said to satisfy conformal restriction if for each conformal embedding $S_{1} \rightarrow S_{2}$, the restriction of $\mu_{S_{2}}$ to $\operatorname{Loop}\left(S_{1}\right)$ equals $\mu_{S_{1}}$; the family is nontrivial if the measure of the set

$$
\{\gamma \in \operatorname{Loop}(\{0<|z|<A\}) \backslash \operatorname{Loop}(\{|z|<a\}): \gamma \text { surrounds } 0\}
$$

is finite and positive, for some $0<a<A$. In [17] Werner proved the following remarkable result.

Theorem 1.1. There exists a nontrivial family of measures $\left\{\mu_{S}\right\}$ on self-avoiding loops on Riemann surfaces which satisfies conformal restriction. This family is unique up to multiplication by an overall positive constant. 
Remark 1.2. In the case of the plane, $S=\mathbb{C}$, this measure is conjectured to be the continuum limit of a (properly weighted) random self-avoiding circular walk (see Section 7.1 of [17]). Kontsevich and Suhov have conjectured that there is a deformation of Werner's (family of) measures to a (family of) measures having values in a determinant line bundle $\operatorname{Det}^{c}$, where $c \leq 1$ is central charge; this deformation is presumably a continuum limit for other statistical mechanical models, and the determinant twist (when $c \neq 0$ ) is essential in understanding how the theory naturally extends to all Riemann surfaces (see [13] and [4]). We refer the reader to the introduction of [17] and Section 6 of [13] for further background and motivation, and [3] for a connection with Schramm-Loewner evolution.

Below we will introduce a normalization which uniquely determines Werner's family of measures (see (1.3)). We will assume this is in force from now on.

Essentially because any self-avoiding loop on a Riemann surface is contained in an embedded annulus, the family $\left\{\mu_{S}\right\}$ is (in principle) uniquely determined by

$$
\mu_{0}:=\left.\mu\right|_{\operatorname{Loop}^{1}(\mathbb{C} \backslash\{0\})}
$$

the restriction of $\mu$ to loops in the plane which surround 0 . The measure $\mu_{0}$ is determined, up to a constant, by the following formula of Werner (see Proposition 3 of [17]):

Theorem 1.3. Suppose that $0 \in U \subset V$, where $U$ and $V$ are bounded simply connected domains in $\mathbb{C}$. Then

$$
\mu_{0}\left(\operatorname{Loop}^{1}(V \backslash\{0\}) \backslash \operatorname{Loop}(U)\right)=c_{\mathrm{W}} \log \left(\left|\phi^{\prime}(0)\right|\right),
$$

where $\phi:(U, 0) \rightarrow(V, 0)$ is a conformal isomorphism.

We will refer to $c_{\mathrm{W}}$ as Werner's constant, which depends on the normalization (1.3). At the present time we can only say that $c_{\mathrm{W}} \geq 1$ (see Section 7.2).

Our purpose is to explore other possible explicit formulas for $\mu_{0}$, especially in terms of welding. To put this in perspective, it is convenient to slightly digress and recall the "fundamental theorem of Welding", and some associated terminology (we recommend [5] as a basic reference).

Theorem 1.4. Suppose that $\sigma$ is a quasisymmetric homeomorphism of $S^{1}$. Then

$$
\sigma=l \circ m a \circ u,
$$

where

$$
u=z\left(1+\sum_{n \geq 1} u_{n} z^{n}\right)
$$

is a univalent holomorphic function in the open unit disk $\Delta$, with quasiconformal extension to $\mathbb{C} \cup\{\infty\}, m \in S^{1}$ is a rotation, $0<a \leq 1$ is a dilation, the mapping inverse to $l$,

$$
L(z)=z\left(1+\sum_{m \geq 1} b_{m} z^{-m}\right)
$$

is a univalent holomorphic function on the open unit disk about infinity, $\Delta^{*}$, with quasiconformal extension to $\mathbb{C} \cup\{\infty\}$, and the compatibility condition

$$
\operatorname{mau}\left(S^{1}\right)=L\left(S^{1}\right)
$$

holds. This factorization is unique. 
Definition 1.5. A homeomorphism $\sigma$ of $S^{1}$ has a triangular factorization (or welding) if $\sigma=$ $l \circ m a \circ u$, where

$$
u(z)=z\left(1+\sum_{n \geq 1} u_{n} z^{n}\right)
$$

is a holomorphic function in $\Delta$ with a continuous extension to a homeomorphism on $D:=$ closure $(\Delta), m \in S^{1}$ is a rotation, $0<a$ is a dilation, the mapping inverse to $l$,

$$
L(z)=z\left(1+\sum_{m \geq 1} b_{m} z^{-m}\right)
$$

is a holomorphic function on $\Delta^{*}$ with a continuous extension to a homeomorphism on $D^{*}=$ $\operatorname{closure}\left(\Delta^{*}\right)$, and the compatibility condition $\operatorname{mau}\left(S^{1}\right)=L\left(S^{1}\right)$ holds.

Quasisymmetric homeomorphisms have unique triangular factorizations. For less regular homeomorphisms, there are additional sufficient conditions for the existence of weldings (see [2] and references, and [1]), but there are many examples of homeomorphisms which do not admit weldings, and weldings which are not unique (see [5]).

Suppose that $\gamma \in \operatorname{Loop}^{1}(\mathbb{C} \backslash\{0\})$. By the Jordan curve theorem the complement of $\gamma$ in $\mathbb{C} \cup\{\infty\}$ has two connected components, $U_{ \pm}$, so that

$$
\mathbb{C} \cup\{\infty\}=U_{+} \sqcup \gamma \sqcup U_{-},
$$

where $0 \in U_{+}$and $\infty \in U_{-}$. There are based conformal isomorphisms

$$
\phi_{+}:(\Delta, 0) \rightarrow\left(U_{+}, 0\right), \quad \phi_{-}:\left(\Delta^{*}, \infty\right) \rightarrow\left(U_{-}, \infty\right) .
$$

The map $\phi_{-}$can be uniquely determined by normalizing the Laurent expansion in $|z|>1$ to be of the form

$$
\phi_{-}(z)=\rho_{\infty}(\gamma) L(z), \quad L(z)=z\left(1+\sum_{n \geq 1} b_{n} z^{-n}\right),
$$

where $\rho_{\infty}(\gamma)>0$ is the transfinite diameter (see Chapters 16 and 17 of [10] for numerous formulas for $\rho_{\infty}$ ). The map $\phi_{+}$can be similarly uniquely determined by normalizing its Taylor expansion to be of the form

$$
\phi_{+}(z)=\rho_{0}(\gamma) u(z), \quad u(z)=z\left(1+\sum_{n \geq 1} u_{n} z^{n}\right)
$$

where $\rho_{0}(\gamma)>0$ is called the conformal radius with respect to 0 . By a theorem of Carathéodory (see Theorem 17.5.3 of [10]), both $\phi_{ \pm}$extend uniquely to homeomorphisms of the closures of their domain and target. This implies that the restrictions $\phi_{ \pm}: S^{1} \rightarrow \gamma$ are topological isomorphisms. Thus there is a well-defined welding map

$$
W: \operatorname{Loop}^{1}(\mathbb{C} \backslash\{0\}) \rightarrow\left\{\sigma \in \operatorname{Homeo}^{+}\left(S^{1}\right): \sigma=l a u\right\} \times \mathbb{R}^{+}: \gamma \mapsto\left(\sigma(\gamma), \rho_{\infty}(\gamma)\right),
$$

where

$$
\sigma(\gamma, z):=\phi_{-}^{-1}\left(\phi_{+}(z)\right)=\text { lau, } \quad a(\gamma)=\frac{\rho_{0}(\gamma)}{\rho_{\infty}(\gamma)}
$$

and $l$ is the inverse mapping for $L$. 


\section{Remarks 1.6.}

(a) To clarify (1.2), the $\sigma$ image of $W$ is by definition the set of homeomorphisms which admit a triangular factorization with rotation $m=1$.

(b) The map $W$ is not $1-1$ because triangular factorization fails (in a dramatic way) to be unique (the source of nonuniqueness: there exist homeomorphisms of the 2-sphere which are conformal off of a Jordan curve, and which are not linear fractional transformations; see $[5])$.

A lofty goal (not in sight) is to calculate, in some explicit way, the image measure $W_{*} \mu_{0}$, and to show that $\mu_{0}$ can be recovered from this image. As we will see in Section 2, conformal invariance implies that

$$
d\left(W_{*} \mu_{0}\right)\left(\sigma, \rho_{\infty}\right)=d \nu_{0}(\sigma) \times \frac{d \rho_{\infty}}{\rho_{\infty}},
$$

where $\nu_{0}$ is an inversion invariant finite measure, which we normalize to have unit mass. This reduces the task of computing $W_{*} \mu_{0}$ to computing the inversion invariant probability measure $\nu_{0}$.

In this paper our first major objective is to show that the measure $\mu_{0}$ is infinitesimally invariant with respect to conformal vector fields, essentially the Virasoro algebra of conformal field theory. This makes essential use of classical variational formulas of Duren and Schiffer [9], which we reformulate in representation theoretic terms for efficient computation. We secondly show how conformal invariance can be used to calculate integrals with respect to the measure $\nu_{0}$ ( $\nu_{0}$ is not itself conformally invariant, so this is a nontrivial step). We thirdly show how these formulas can be used to calculate the joint moments for the coefficients of $u$. Since these coefficients are bounded, these moments (in principle) determine the joint distributions for the coefficients. This yields an alternate proof of the uniqueness of Werner's measure. This is also potentially interesting because a sufficiently explicit calculation of the individual moments for $u_{n}$ could yield a probabilistic proof of the Bieberbach conjecture/de Branges theorem (as pointed out by a referee, one must also show the measure $\nu_{0}$ has dense support in a suitable sense). Our current procedure (which we have implemented numerically) has the virtue that it in principle systematically calculates all joint moments; it has the drawback that to obtain a general moment for $u_{N}$, it has to calculate on the order of $p(N)$ joint moments for all $u_{n}$ with $n<N$, where $p(N)$ is the partition function (which grows very rapidly). In any event a certain fraction of the moments turn out to have remarkably simple expressions; for example:

\section{Theorem 1.7.}

$$
\int\left|u_{n}\right|^{2} d \nu_{0}=\frac{1}{n+1}
$$

The coefficients of $u$ are well-known to be functionally dependent in a very complicated way (see Chapter 11 of [8]). For this reason it seems unlikely that one could calculate $\nu_{0}$ in an explicit way in terms of these coordinates. For this reason it is important to consider other quantities (and coordinates) associated with the welding homeomorphism $\sigma$. For various reasons (see Remarks 1.9 below), it is of special interest to calculate the "diagonal distribution", i.e. the distribution for $a$ in the triangular factorization $\sigma=l a u$.

Conjecture 1.8. If $\nu_{0}$ is normalized to be a probability measure, then

$$
\nu_{0}(\{\sigma: \exp (-x) \leq a(\sigma) \leq 1\})=\exp \left(-\frac{\beta_{0}}{x}\right), \quad x>0
$$

for some constant $\beta_{0}<\frac{5 \pi^{2}}{4}$. 


\section{Remarks 1.9.}

(a) The motivating idea is to show that the Laplace transform of the diagonal distribution for $\nu_{0}$ satisfies a differential equation, using the infinitesimal conformal invariance of $\mu_{0}$.

(b) This conjecture is closely related to Proposition 18 in [17], concerning the measure of the set of nontrivial loops in a finite type annulus, for which there is an explicit conjecture due to Cardy (see Section 7).

(c) There is a natural generalization of this conjecture to the deformation of Werner's measure which is conjectured to exist in [13] (see Section 7.4 and see [4] for recent progress on this conjecture). Our hope is that this extended conjecture might be useful in proving existence of this deformation.

To close this introduction, we mention one obvious coordinate which should be investigated. For a homeomorphism $\sigma$ of $S^{1}$, write

$$
\sigma\left(e^{i \theta}\right)=e^{i \Sigma(\theta)}
$$

where the lift $\Sigma$ is a homeomorphism of $\mathbb{R}$ satisfying $\Sigma(\theta+2 \pi)=\Sigma(\theta)+2 \pi ; \Sigma$ is determined modulo $2 \pi \mathbb{Z}$. The $\nu_{0}$ distribution for $\sigma$ is completely determined by the distributional derivative,

$$
\frac{1}{2 \pi} d \Sigma
$$

which we view as a probability measure on $S^{1}$.

Verblunsky discovered a remarkable parameterization of probability measures on $S^{1}$. To state the gist of the result simply (following [16]), let $\operatorname{Prob}^{\prime}\left(S^{1}\right)$ denote the set of probability measures which are nontrivial, in the sense that their support is not a finite set.

Theorem 1.10. The following map induces a bijective correspondence:

$$
\operatorname{Prob}^{\prime}\left(S^{1}\right) \rightarrow \prod_{n=0}^{\infty} \Delta: \omega \rightarrow\left(\alpha_{n}\right),
$$

where $p_{0}=1, p_{1}=z-\overline{\alpha_{1}}, \ldots$ are the monic orthogonal polynomials with respect to $\omega$, and $\alpha_{n}=-\overline{p_{n+1}(0)}$.

It is very striking that the image of this correspondence is a product space, i.e. the $\alpha_{n}$ are functionally independent, in sharp contrast to the coefficients $u_{n}$. This suggests the following naive

Question 1.11. Are the Verblunsky coefficients

$$
\left(\alpha_{n}\right) \in \prod_{n=0}^{\infty} \Delta
$$

independent random variables with respect to $\nu_{0}$ ?

We have basically failed in trying to investigate this question numerically. 


\subsection{Outline of the paper}

In Section 2 we prove some basic facts about the welding map $W$. In Section 3 we recall some classical variational formulas of Duren and Schiffer. In Section 4 we discuss the infinitesimal action from a representation theoretic point of view, and we recast the Duren-Schiffer formulas in terms of generating functions, using a stress-energy tensor formulation common in conformal field theory. In Section 5 we establish the version of infinitesimal conformal invariance of $\mu_{0}$ needed for our purposes. In Section 6 we apply this to compute moments of the coefficients of $u$, and to give an alternate proof of the uniqueness of Werner's family of measures. In Section 7 we discuss the relation between the diagonal distribution conjecture and Proposition 18 of [17], and outline a strategy for a proof; we also briefly indicate how the conjecture generalizes to the deformation which is conjectured by Kontsevich and Suhov to exist in [13].

\subsection{Notations and conventions}

Given a complex number $z$, we often write $z^{*}$ for the complex conjugate, especially when $z$ is represented by a complicated expression.

Given a Laurent expansion $f(z)=\sum f_{n} z^{n}$, we write $f^{*}(z)=\sum\left(f_{n}\right)^{*} z^{-n}$ (for $z \in S^{1}$, $\left.f^{*}(z)=f(z)^{*}\right)$. We also write $f_{-}(z)=\sum_{n<0} f_{n} z^{n}, f_{+}(z)=\sum_{n \geq 0} f_{n} z^{n}, f_{++}(z)=\sum_{n>0} f_{n} z^{n}$, and $f_{-1}=\operatorname{Res}(f(z), z=0)$.

Throughout this paper, we view vector fields on a manifold as the Lie algebra of diffeomorphisms of the manifold; the induced bracket is the negative of the usual bracket obtained by viewing vector fields as derivations of functions on the manifold.

\section{The welding map}

In this section we consider the welding map (1.2).

\section{Proposition 2.1.}

(a) The distributions for $\rho_{0}$ and $\rho_{\infty}$ are invariant with respect to dilation, i.e. equivalent to Haar measure for $\mathbb{R}^{+}$.

(b) $d\left(W_{*} \mu_{0}\right)\left(\sigma, \rho_{\infty}\right)=d \nu_{0}(\sigma) \times \frac{d \rho_{\infty}}{\rho_{\infty}}$, where $\nu_{0}$ is a finite measure (which we will normalize to have unit mass).

(c) The measure $d \nu_{0}(\sigma)$ is inversion invariant and invariant with respect to conjugation by $C: z \mapsto z^{*}$.

(d) The measure $d \nu_{0}(\sigma)$ is supported on $\sigma$ having triangular factorization $\sigma=$ lau, i.e. $m=1$.

(e) For any $\gamma \in \operatorname{Loop}^{1}(\mathbb{C} \backslash\{0\})$,

$$
a(\sigma(\gamma))=\left(\frac{1-\sum_{m=1}^{\infty}(m-1)\left|b_{m}\right|^{2}}{1+\sum_{n=1}^{\infty}(n+1)\left|u_{n}\right|^{2}}\right)^{1 / 2} \leq 1
$$

where $u$ and $L$ are written as in Definition 1.5.

(f) The welding map is equivariant with respect to rotations in the sense that

$$
\sigma(\operatorname{Rot}(\theta)(\gamma))=\operatorname{Rot}(\theta) \circ \sigma(\gamma) \circ \operatorname{Rot}(\theta)^{-1}
$$


Proof. We first claim that

$$
\left\{\gamma \in \operatorname{Loop}^{1}(\mathbb{C} \backslash\{0\}): r<\rho_{\infty}(\gamma)<R\right\} \subset \operatorname{Loop}^{1}(\{|z|<4 R\}) \backslash \operatorname{Loop}^{1}(\{r<|z|\}) .
$$

The inequality $r<\rho_{\infty}(\gamma)$ implies that $\gamma$ cannot be contained in $\{|z|<r\}$. In general $\rho_{\infty}(\gamma)=$ $\rho_{0}\left(\frac{1}{\gamma}\right)^{-1}$. Thus if $\rho_{\infty}(\gamma)<R$, then $\frac{1}{R}<\rho_{0}\left(\frac{1}{\gamma}\right)$. The Koebe one-quarter theorem implies that $\frac{1}{\gamma} \subset\left\{\frac{1}{4 R}<|z|\right\}$. Thus $\gamma$ is in the ball of radius $4 R$. This proves the claim.

By conformal invariance and the nontriviality assumption of Werner, the set of loops (surrounding zero) with $r<\rho_{\infty}<R$ has $\mu_{0}$ finite measure, for any $r<R$. This implies that there is a essentially unique disintegration of $\mu_{0}$ of the form

$$
d \mu_{0}(\gamma)=\int_{\rho_{\infty}=0}^{\infty} d \mu_{\rho_{\infty}}(\sigma) d \omega\left(\rho_{\infty}\right)
$$

where the fiber measures are probability measures.

The invariance of $\mu_{0}$ with respect to dilation, $\gamma \rightarrow \rho \gamma$, implies that the $\rho_{\infty}$ distribution $\omega$ is also dilation invariant, i.e. it is a Haar measure for $\mathbb{R}^{+}$. The invariance of $\mu_{0}$ with respect to $z \rightarrow \frac{1}{z}$ implies that the same is true for $\rho_{0}$. This proves (a).

Since $\mu_{0}$ is determined up to multiplication by a constant, we can suppose that

$$
d \omega\left(\rho_{\infty}\right)=\frac{d \rho_{\infty}}{\rho_{\infty}}
$$

The action by dilation transports one fiber to another. Hence dilation invariance also implies that all the fiber measures are the same. This implies that $W_{*} \mu_{0}$ is a product measure, as claimed in part (b).

For part (c), we first use the invariance of $\mu_{0}$ with respect to $z \rightarrow \frac{1}{z^{*}}$, which maps $\gamma$ to $\frac{1}{\gamma^{*}}$ :

$$
\begin{array}{ll}
\phi_{+}\left(\frac{1}{\gamma^{*}}\right)(z)=\frac{1}{\phi_{-}(\gamma)\left(\frac{1}{z^{*}}\right)^{*}}, & |z|<1, \\
\phi_{-}\left(\frac{1}{\gamma^{*}}\right)(z)=\frac{1}{\phi_{+}(\gamma)\left(\frac{1}{z^{*}}\right)^{*}}, & |z|>1,
\end{array}
$$

and

$$
\phi_{-}\left(\frac{1}{\gamma^{*}}\right)^{-1}(w)=\frac{1}{\left(\phi_{+}(\gamma)^{-1}\left(\frac{1}{w^{*}}\right)\right)^{*}} .
$$

Thus

$$
\begin{aligned}
\sigma\left(\frac{1}{\gamma^{*}}\right)(z) & =\phi_{-}\left(\frac{1}{\gamma^{*}}\right)^{-1} \circ \phi_{+}\left(\frac{1}{\gamma^{*}}\right)(z)=\phi_{-}\left(\frac{1}{\gamma^{*}}\right)^{-1}\left(\frac{1}{\phi_{-}(\gamma)\left(\frac{1}{z^{*}}\right)^{*}}\right) \\
& =\frac{1}{\left(\phi_{+}(\gamma)^{-1}\left(\phi_{-}(\gamma)\left(\frac{1}{z^{*}}\right)\right)^{*}\right.}=\frac{1}{\sigma^{-1}(\gamma)\left(\frac{1}{z^{*}}\right)^{*}}=\sigma(\gamma)^{-1}(z) .
\end{aligned}
$$

This implies the invariance of $\nu_{0}$ with respect to inversion.

The measure $\mu_{0}$ is also invariant with respect to $C: z \mapsto z^{*}$. In this case

$$
\phi_{ \pm}\left(\gamma^{*}\right)=C \circ \phi_{ \pm}(\gamma) \circ C
$$

This implies that $\nu_{0}$ is invariant with respect to conjugation by $C$. This proves (c).

Part (d) is obvious. 
For part (e) (essentially the well-known area theorem from the theory of univalent functions), the main point is that

$$
a u(\Delta)=\mathbb{C} \backslash L\left(D^{*}\right) .
$$

For sufficiently smooth $\gamma$

$$
\operatorname{Area}(u(\Delta))=\frac{1}{2 i} \int_{\gamma} d \bar{t} \wedge d t=\frac{1}{2 i} \int_{S^{1}} \bar{u} d u=\pi\left(1+\sum_{n=1}^{\infty}(n+1)\left|u_{n}\right|^{2}\right)
$$

and

$$
\operatorname{Area}\left(\mathbb{C} \backslash L\left(\Delta^{*}\right)\right)=\frac{1}{2 i} \int_{\gamma} \bar{t} d t=\frac{1}{2 i} \int_{S^{1}} \bar{L} d L=\pi\left(1-\sum_{m=1}^{\infty}(m-1)\left|b_{m}\right|^{2}\right) .
$$

By continuity of measure, these formulas hold for all $\gamma$. This implies part (e).

Part (f) follows from

$$
\phi_{ \pm}(\operatorname{Rot}(\theta)(\gamma))=\operatorname{Rot}(\theta) \circ \phi_{ \pm} \circ \operatorname{Rot}(\theta)^{-1} .
$$

\section{Remarks 2.2.}

(a) In connection with part (c), in general, if a homeomorphism $\sigma$ has a triangular factorization lmau, then $\sigma^{-1}$ has a triangular factorization with

$$
\begin{aligned}
& u\left(\sigma^{-1}\right)(z)=\frac{1}{L\left(\frac{1}{z^{*}}\right)^{*}}, \quad l\left(\sigma^{-1}\right)(z)=\frac{1}{U\left(\frac{1}{z^{*}}\right)^{*}}, \\
& m\left(\sigma^{-1}\right)=m(\sigma)^{*}, \quad a\left(\sigma^{-1}\right)=a(\sigma) .
\end{aligned}
$$

In particular inversion stabilizes the set of $\sigma$ having triangular factorization with $m=1$.

(b) In connection with part (f), equivariance with respect to rotations, see Section 3.2 below.

\subsection{Unresolved foundational issues}

Theorem 1.4 implies that $W$ induces a bijection

$$
W: \operatorname{QuasiCircles}^{1}(\mathbb{C} \backslash\{0\}) \leftrightarrow\left\{\sigma \in \mathrm{QS}\left(S^{1}\right): \sigma=l a u\right\} \times \mathbb{R}^{+},
$$

where a quasicircle is a Jordan curve which admits a parameterization by the restriction to $S^{1}$ of a quasiconformal homeomorphism of $\mathbb{C} \cup\{\infty\}$.

\section{Conjecture 2.3.}

(a) $\mu_{0}$ has measure zero on quasicircles.

(b) $W$ is $1-1$ on a set of full $\mu_{0}$ measure.

(c) Almost surely with respect to $\nu_{0}, \sigma$ has a unique triangular factorization (with $m=1$ ).

This kind of issue is addressed in [1] and [2].

In this paper we will need to avoid these unresolved issues. In particular, because we do not know that $u$ is determined by $\sigma$ (in an almost sure sense), in the remainder of this paper, we will implicitly view $\nu_{0}$ as a measure on $\{u\}$. Thus in place of (b) of Proposition 2.1, we will use the following decomposition, which is proved in exactly the same way.

\section{Proposition 2.4.}

$$
d \mu_{0}\left(u, \rho_{\infty}\right)=d \nu_{0}(u) \times \frac{d \rho_{\infty}}{\rho_{\infty}},
$$

where $\nu_{0}$ is a finite measure (which we will normalize to have unit mass). 


\section{$3 \quad$ Variational formulas}

Since the measure $\mu_{0}$ has a local form of conformal invariance, it is natural to suspect that there are senses in which the measure is infinitesimally conformally invariant. For this reason we need to consider how $\phi_{ \pm}$vary when the curve $\gamma$ is varied by a local deformation $z \mapsto z+\epsilon v(z)$, where $v(z)$ is holomorphic in $\mathbb{C} \backslash\{0\}$. This deformation corresponds to a real vector field

$$
\vec{v}=v_{1} \frac{\partial}{\partial x}+v_{2} \frac{\partial}{\partial y}
$$

where $v=v_{1}+i v_{2}$. Let $\overrightarrow{\mathcal{W}}$ denote the real Lie algebra of all such vector fields, where $v(z)$ has a finite Laurent expansion.

For technical reasons, we distinguish $\overrightarrow{\mathcal{W}}$ from the Witt algebra $\mathcal{W}$, which consists of holomorphic vector fields $v(z) \frac{\partial}{\partial z}$, where again $v(z)$ has a finite Laurent expansion. The Witt algebra is a complex Lie algebra. It is spanned over $\mathbb{C}$ by the vector fields

$$
L_{n}=-z^{n+1} \frac{\partial}{\partial z}, \quad n \in \mathbb{Z}
$$

with bracket

$$
\left[L_{n}, L_{m}\right]=(m-n) L_{n+m}
$$

$W^{*}$ consists of antiholomorphic vector fields. It is spanned by $\left\{\bar{L}_{n}: n \in \mathbb{Z}\right\}$.

The precise relationship between $\overrightarrow{\mathcal{W}}$ and $\mathcal{W}$ is that there is a real embedding

$$
\overrightarrow{\mathcal{W}} \rightarrow \mathcal{W} \oplus \mathcal{W}^{*}: \vec{v}=v_{1} \frac{\partial}{\partial x}+v_{2} \frac{\partial}{\partial y} \rightarrow v(z) \frac{\partial}{\partial z}+v^{*}(z) \frac{\partial}{\partial \bar{z}} .
$$

Loosely speaking, $\overrightarrow{\mathcal{W}}$ is the Witt algebra considered as a real Lie algebra (see [7, p. 115]). The reason for maintaining a distinction is that the variational formulas below will naturally define a real representation of the real Lie algebra $\overrightarrow{\mathcal{W}}$. However it is convenient to express this representation in terms of an associated complex representation of $\mathcal{W}$. We will write the map (3.1) as

$$
\vec{L} \leftrightarrow(L, \bar{L}) .
$$

In particular

$$
\vec{L}_{n} \leftrightarrow\left(L_{n}, \bar{L}_{n}\right) \quad \text { and } \quad \overrightarrow{i L}_{n} \leftrightarrow\left(i L_{n}, \overline{i L}_{n}\right)=\left(i L_{n},-i\left(\bar{L}_{n}\right)\right) .
$$

\subsection{Variational formulas, I}

$\overrightarrow{\mathcal{W}}$ can be viewed as a Lie algebra of vector fields on $\operatorname{Loop}^{1}(\mathbb{C} \backslash\{0\})$, where by definition a vector field on a self-avoiding loop is simply a $\mathbb{R}^{2}$-valued vector field along the loop (the degree of smoothness of a loop is not relevant here). In particular

$$
\left.\vec{L}_{n}\right|_{\gamma}=\left.\frac{d}{d t} \exp \left(t \vec{L}_{n}\right)(\gamma)\right|_{t=0}
$$

and similarly for $\overrightarrow{i L}_{n}$. The corresponding actions on a function of $\gamma$ are given by

$$
\vec{L}_{n} \cdot F(\gamma)=\left.\frac{d}{d t}\right|_{t=0} F\left(\exp \left(-t \vec{L}_{n}\right)(\gamma)\right)=\left.\frac{d}{d t}\right|_{t=0} F\left(\gamma+t \gamma^{n+1}\right)
$$

where in the last line we have implicitly chosen a parameterization for $\gamma$, and similarly for $\overrightarrow{i L}_{n}$. 
When $n \geq-1, L_{n}=-z^{n+1} \frac{\partial}{\partial z}$ is regular at $z=0$. In this case it is very easy to find the variations of $\phi_{+}$with respect to $\vec{L}_{n}$ and $\overrightarrow{i L}_{n}$.

\section{Proposition 3.1.}

(a) For $n \geq 0, \vec{L}_{n} \phi_{+}=\phi_{+}^{n+1}$. In particular

$$
\vec{L}_{0} \rho_{0}=\rho_{0}, \quad \text { and } \quad \vec{L}_{0} u_{k}=0, \quad k \geq 1,
$$

and for $n>0$

$$
\vec{L}_{n} \rho_{0}=0, \quad \vec{L}_{n} u_{k}=0 \quad k<n, \quad \vec{L}_{n} u_{n}=\rho_{0}^{n},
$$

and for $n<k$

$$
\vec{L}_{n} u_{k}=\rho_{0}^{n} p_{k-n}^{(n+1)}\left(u_{1}, \ldots, u_{k-n}\right),
$$

where

$$
\left(1+u_{1} z+u_{2} z^{2}+\cdots\right)^{n+1}=\sum_{l=0}^{\infty} p_{l}^{(n+1)}\left(u_{1}, \ldots, u_{l}\right) z^{l}
$$

(b) $\vec{L}_{-1} \phi_{+}=1+u^{\prime}(z)\left(-1+\left(u_{1}-u_{1}^{*}\right) z+z^{2}\right)$.

(c) For $n>0,\left(\overrightarrow{i L}_{n}\right) \phi_{+}=i \phi_{+}^{n+1}$, and $\left(\overrightarrow{i L}_{0}\right) \phi_{+}=i\left(\phi_{+}-z \phi_{+}^{\prime}\right)$.

(d) $\left(\overrightarrow{i L}_{-1}\right) \phi_{+}=i\left(1+u^{\prime}(z)\left(-1+\left(u_{1}+u_{1}^{*}\right) z-z^{2}\right)\right)$.

Proof. $\vec{L}_{0}$ is infinitesimal dilation. In this case the formulas in part (a) are obvious, because $\sigma(\gamma)$ is unchanged when $\gamma$ is dilated.

For $n \geq-1$ and $\epsilon$ sufficiently small, a uniformization for the region inside $\gamma+\epsilon \gamma^{n+1}$ is the composition

$$
\phi_{+}+\epsilon\left(\phi_{+}\right)^{n+1} .
$$

This uniformization has to be composed with a linear fractional transformation to obtain the correct normalization. Consequently

$$
\phi_{+}\left(\gamma+\epsilon \gamma^{n+1}\right)(z)=\left(\phi_{+}+\epsilon\left(\phi_{+}\right)^{n+1}\right)\left(\frac{\lambda(\epsilon) z+\omega(\epsilon)}{1+\omega(\epsilon)^{*} \lambda(\epsilon) z}\right)
$$

where $\lambda$ (having unit norm) and $\omega$ are determined by the conditions that this uniformization vanishes at $z=0$ and has positive derivative at $z=0$.

Suppose $n \geq 0$. In this case the linear fractional transformation is the identity for all $\epsilon$. This implies part (a).

Part (b), when $n+1=0$, is slightly more involved. In this case

$$
\begin{aligned}
\left(\vec{L}_{-1} \phi_{+}\right)(z) & =\left.\frac{d}{d \epsilon}\right|_{\epsilon=0}\left(\phi_{+}\left(\frac{\lambda(\epsilon) z+\omega(\epsilon)}{1+\omega(\epsilon)^{*} \lambda(\epsilon) z}\right)+\epsilon\right) \\
& =\phi_{+}^{\prime}(z)\left(\dot{\lambda}(0) z+\dot{\omega}(0)-z \dot{\omega}(0)^{*} z\right)+1 .
\end{aligned}
$$


To calculate the derivatives at zero, we use the normalizations for the mapping (3.2). Because 0 is mapped to zero,

$$
\epsilon+\phi_{+}(\omega(\epsilon))=0
$$

This implies

$$
\omega(\epsilon)=\phi_{+}^{-1}(-\epsilon) \quad \text { and } \quad \dot{\omega}(0)=-\rho_{0}^{-1} .
$$

Secondly the derivative of the map (3.2) at $z=0$ must be positive. Thus

$$
\phi_{+}^{\prime}(\omega(\epsilon)) \lambda(\epsilon)\left(1-|\omega(\epsilon)|^{2}\right)>0
$$

and (because $\lambda$ has unit norm)

$$
\lambda(\epsilon)^{-1}=\exp \left(i \operatorname{Im}\left(\log \left(\phi_{+}^{\prime}(\omega(\epsilon))\right)\right)\right) .
$$

This implies

$$
\dot{\lambda}(0)=-i \operatorname{Im}\left(\frac{\phi_{+}^{\prime \prime}(\omega(0)) \dot{\omega}(0)}{\phi_{+}^{\prime}(\omega(0))}\right)=-2 i \operatorname{Im}\left(\frac{u_{1}}{\rho_{0}}\right) .
$$

Plugging these derivatives into (3.3) yields

$$
\vec{L}_{-1} \phi_{+}=1+\rho_{0}\left(1+2 u_{1} z+3 u_{2} z^{2}+\cdots\right)\left(\frac{u_{1}-u_{1}^{*}}{\rho_{0}} z-\frac{1}{\rho_{0}}+\frac{1}{\rho_{0}} z^{2}\right) .
$$

This implies part (b).

Parts (c) and (d) are similar. For part (c), when $n=0$, note that

$$
\left(\exp \left(i \theta \vec{L}_{0}\right) \phi_{+}\right)(z)=e^{i \theta} \phi_{+}\left(e^{-i \theta} z\right)
$$

so that

$$
\exp \left(i \theta \vec{L}_{0}\right) \rho_{0}=\rho_{0} \quad \text { and } \quad \exp \left(i \theta \vec{L}_{0}\right) u_{k}=e^{-i k \theta} u_{k}
$$

For part (d), when $n+1=0$,

$$
\phi_{+}(\gamma+i \epsilon)(z)=\phi_{+}\left(\frac{\lambda(\epsilon) z+\omega(\epsilon)}{1+\omega(\epsilon)^{*} \lambda(\epsilon) z}\right)+i \epsilon
$$

and

$$
\begin{aligned}
\left(\overrightarrow{i L}_{-1} \phi_{+}\right)(z) & =\left.\frac{d}{d \epsilon}\right|_{\epsilon=0}\left(\phi_{+}\left(\frac{\lambda(\epsilon) z+\omega(\epsilon)}{1+\omega(\epsilon)^{*} \lambda(\epsilon) z}\right)+i \epsilon\right) \\
& =\phi_{+}^{\prime}(z)\left(\dot{\lambda}(0) z+\dot{\omega}(0)-z \dot{\omega}(0)^{*} z\right)+i .
\end{aligned}
$$

To calculate the derivatives at zero, we use the normalizations for the mapping (3.4). Because 0 is mapped to zero,

$$
i \epsilon+\phi_{+}(\omega(\epsilon))=0 .
$$

This implies

$$
\omega(\epsilon)=\phi_{+}^{-1}(-i \epsilon) \quad \text { and } \quad \dot{\omega}(0)=-i \rho_{0}^{-1} .
$$


Secondly the derivative of the map (3.4) at $z=0$ must be positive. Thus

$$
\phi_{+}^{\prime}(\omega(\epsilon)) \lambda(\epsilon)\left(1-|\omega(\epsilon)|^{2}\right)>0
$$

and (because $\lambda$ has unit norm)

$$
\lambda(\epsilon)^{-1}=\exp \left(i \operatorname{Im}\left(\log \left(\phi_{+}^{\prime}(\omega(\epsilon))\right)\right)\right) .
$$

This implies

$$
\dot{\lambda}(0)=-i \operatorname{Im}\left(\frac{\phi_{+}^{\prime \prime}(\omega(0)) \dot{\omega}(0)}{\phi_{+}^{\prime}(\omega(0))}\right)=2 i \operatorname{Im}\left(\frac{i u_{1}}{\rho_{0}}\right)=i \frac{u_{1}+u_{1}^{*}}{\rho_{0}} .
$$

Plugging these derivatives into (3.5) yields

$$
\overrightarrow{i L}_{-1} \phi_{+}=i+\rho_{0}\left(1+2 u_{1} z+3 u_{2} z^{2}+\cdots\right)\left(\frac{i\left(u_{1}+u_{1}^{*}\right)}{\rho_{0}} z-\frac{i}{\rho_{0}}-\frac{i}{\rho_{0}} z^{2}\right) .
$$

This implies part (d).

\section{2 (Lack of) Equivariance for $W$}

We have already observed that the welding map is equivariant with respect to the actions of rotation of loops and conjugation of homeomorphisms; see (f) of Proposition 2.1.

Given $|w|<1$, define $\phi_{1}(w) \in \operatorname{PSU}(1,1)$ (viewed as the group of automorphisms of the Riemann sphere which stabilize the circle) by

$$
\phi_{1}(w ; z)=\frac{z+\bar{w}}{1+w z} .
$$

Proposition 3.2. Suppose that $\gamma \in \operatorname{Loop}^{1}(\mathbb{C} \backslash\{0\})$ such that $\phi_{1}(\epsilon, \gamma) \in \operatorname{Loop}^{1}(\mathbb{C} \backslash\{0\})$. Then to first order in $\epsilon$

(a) $\phi_{+}\left(\phi_{1}(\epsilon, \gamma)\right)=\phi_{1}(\epsilon) \circ \phi_{+}(\gamma) \circ \phi_{1}\left(-\epsilon / \rho_{0}\right) \circ \exp \left(2 i \operatorname{Im}\left(u_{1} \bar{\epsilon}\right) / \rho_{0}\right)$,

(b) $\phi_{-}\left(\phi_{1}(\epsilon, \gamma)\right)=\phi_{1}(\epsilon) \circ \phi_{-}(\gamma) \circ \phi_{1}\left(-\rho_{\infty} \epsilon\right) \circ \exp \left(-2 i \rho_{\infty} \operatorname{Im}\left(b_{1} \epsilon\right)\right)$,

(c) $\sigma\left(\phi_{1}(\epsilon, \gamma)\right)=\exp \left(-2 i \rho_{\infty} \operatorname{Im}\left(b_{1} \epsilon\right)\right) \circ \phi_{1}\left(\rho_{\infty} \epsilon\right) \circ \sigma(\gamma) \circ \phi_{1}\left(-\epsilon / \rho_{0}\right) \circ \exp \left(2 i \operatorname{Im}\left(u_{1} \bar{\epsilon}\right) / \rho_{0}\right)$.

Remark 3.3. The formula in (c) illustrates how the welding map is trying (with limited success) to intertwine the action of $\operatorname{PSU}(1,1)$ on loops with its action by conjugation on the welding homeomorphism.

Proof. A uniformization for the region inside $\gamma(\epsilon)$ is the composition

$$
\phi_{1}\left(\epsilon, \phi_{+}(\gamma)(z)\right)
$$

This uniformization has to be precomposed with a linear fractional transformation to obtain the correct normalization. Consequently

$$
\phi_{+}(\gamma(\epsilon))=\phi_{1}(\epsilon) \circ \phi_{+}(\gamma) \circ \Phi_{1}(\epsilon),
$$

where

$$
\Phi_{1}(\epsilon, z)=\frac{\lambda(\epsilon) z+\bar{\omega}(\epsilon)}{1+\omega(\epsilon) \lambda(\epsilon) z}
$$


and $\lambda$ (having unit norm) and $\omega$ are determined by the conditions that this uniformization vanishes at $z=0$ and has positive derivative at $z=0$.

The first condition implies

$$
\Phi_{1}(\epsilon, 0)=\bar{\omega}(\epsilon)=\phi_{+}^{-1}(-\epsilon)=-\frac{\epsilon}{\rho_{0}}+O\left(\epsilon^{2}\right),
$$

in particular $\omega^{\prime}(0)=-\rho_{0}^{-1}$. Note that for $\Phi_{1}$ to exist, $-\epsilon$ must be in $U_{+}$. The second condition

$$
\phi_{1}(\epsilon)^{\prime}\left[\phi_{+} \circ \Phi_{1}(0)\right] \phi_{+}^{\prime}\left[\Phi_{1}(0)\right] \Phi_{1}^{\prime}(0)>0
$$

is equivalent to

$$
\phi_{1}(\epsilon)^{\prime}\left[\phi_{+}(\bar{\omega}(\epsilon)] \phi_{+}^{\prime}[\bar{\omega}(\epsilon)] \lambda\left(1-|\omega|^{2}\right)>0\right.
$$

or

$$
\bar{\lambda}(\epsilon)=\frac{\phi_{1}(\epsilon)^{\prime}\left[\phi_{+}(\bar{\omega}(\epsilon)] \phi_{+}^{\prime}[\bar{\omega}(\epsilon)]\right.}{\mid \phi_{1}(\epsilon)^{\prime}\left[\phi_{+}(\bar{\omega}(\epsilon)] \phi_{+}^{\prime}[\bar{\omega}(\epsilon)] \mid\right.} .
$$

Use

$$
\begin{aligned}
& \phi_{1}(\epsilon)^{\prime}(z)=\frac{1-|\epsilon|^{2}}{(1+\epsilon z)^{2}}, \\
& \phi_{+}(\bar{\omega}(\epsilon))=\rho_{0} \bar{\omega}(\epsilon)+O\left(\epsilon^{2}\right)=-\epsilon+O\left(\epsilon^{2}\right), \\
& \phi_{1}(\epsilon)^{\prime}\left(\phi_{+}(\bar{\omega}(\epsilon))=\frac{1-|\epsilon|^{2}}{\left(1+\epsilon\left(-\frac{\epsilon}{\rho_{0}}+O\left(\epsilon^{2}\right)\right)\right)^{2}}=1+O\left(\epsilon^{2}\right),\right. \\
& \phi_{+}^{\prime}[\bar{\omega}(\epsilon)]=\rho_{0}+2 \rho_{0} u_{1} \bar{\omega}(\epsilon)=\rho_{0}-2 u_{1} \epsilon+O\left(\epsilon^{2}\right) .
\end{aligned}
$$

Putting everything together

$$
\bar{\lambda}(\epsilon)=\frac{\left(1+\epsilon^{2}+\cdots\right)\left(\rho_{0}-2 u_{1} \epsilon+\cdots\right)}{\left|\left(1+\epsilon^{2}+\cdots\right)\left(\rho_{0}-2 u_{1} \epsilon+\cdots\right)\right|}=1-2 \frac{\left(u_{1}-\overline{u_{1}}\right) \epsilon}{\rho_{0}}+O\left(\epsilon^{2}\right) .
$$

This implies the formula in (a).

In a similar way

$$
\phi_{-}\left(\phi_{1}(\epsilon ; \gamma)\right)=\phi(\epsilon) \circ \phi_{-}(\gamma) \Psi_{1}
$$

and one precedes as before. This leads to (b) and (c).

\subsection{Variational formulas, II}

It is far more difficult to calculate $\vec{L}_{-n} \phi_{+}$for $n>1$. In this case $z^{-n+1}$ is regular at $z=\infty$. This is the situation considered in [9], with slight modifications. The following statement is essentially equation (17) in [9].

Proposition 3.4. Suppose that $n \geq 1$. Then

(a) $\vec{L}_{-n} \rho_{0}=\rho_{0}^{-n+1} \operatorname{Re}\left(P_{n}\left(u_{1}, \ldots, u_{n}\right)\right)$, where

$$
P_{n}\left(u_{1}, \ldots, u_{n}\right)=\operatorname{Res}\left(\left(\frac{U^{\prime}(t)}{U(t)}\right)^{2} t^{-n+1}, t=0\right)
$$

(as always, $U$ is the mapping inverse to $u$ ). If $\operatorname{deg}\left(u_{j}\right)=j$, then $P_{n}$ is a homogeneous polynomial of degree $n$. 
(b) For $k \geq 1$

$$
\vec{L}_{-n} u_{k}=\rho_{0}^{-n}\left(k u_{k} \operatorname{Re}\left(B_{0}\right)+\sum_{m=1}^{k}(k+1-m) u_{k-m}\left(B_{-m}+\overline{B_{m}}\right)\right),
$$

where

$$
B_{m}=\operatorname{Res}\left(\left(\frac{U^{\prime}(t)}{U(t)}\right)^{2} U(t)^{m} t^{-n+1}, t=0\right)=\operatorname{Res}\left(\frac{u(z)^{-n+1}}{u^{\prime}(z)} z^{m-2}, z=0\right) .
$$

(c) $\overrightarrow{i L}_{-n} \rho_{0}=-\rho_{0}^{-n+1} \operatorname{Im}\left(P_{n}\left(u_{1}, \ldots, u_{n}\right)\right)$.

(d) For $k \geq 1$

$$
\overrightarrow{i L}_{-n} u_{k}=-\rho_{0}^{-n}\left(k u_{k} \operatorname{Im}\left(B_{0}\right)+\sum_{m=1}^{k}(k+1-m) u_{k-m} i\left(-B_{-m}+\overline{B_{m}}\right)\right) .
$$

Remarks 3.5. (a) It is natural to restate the relationship between the $P_{n}$ and $U$ in terms of quadratic differentials

$$
(\partial \log (U(t)))^{2}=\sum_{n=0}^{\infty} P_{n}(u) t^{n}\left(\frac{d t}{t}\right)^{2},
$$

where $t=u(z), z=U(t)$. In Section 7 it will be convenient to rewrite this as

$$
\left(\partial \log \left(\phi_{+}^{-1}(t)\right)\right)^{2}=\sum_{n=0}^{\infty} \rho_{0}^{-n} P_{n}(u) t^{n}\left(\frac{d t}{t}\right)^{2}
$$

and to set $P_{n}\left(\phi_{+}\right)=\rho_{0}^{-n} P_{n}(u)$. Hopefully this will not cause any confusion.

(b) Similarly the residue formula for $B_{m}$ is naturally understood as the integral over $\gamma$ of the natural pairing of the holomorphic vector field $-v(t) \frac{d}{d t}$ and the holomorphic quadratic differential $U(t)^{m}(\partial \log (U(t)))^{2}$.

For later reference we note some elementary properties of the polynomials $P_{n}$.

\section{Proposition 3.6.}

(a) $P_{n}(u)$ is a homogeneous polynomial in $u_{1}, \ldots, u_{n}$ of degree $n$, where $\operatorname{deg}\left(u_{j}\right)=j$, with integer coefficients.

(b) $P_{n}(u)=-2 n u_{n}+$ terms involving $u_{1}, \ldots, u_{n-1}$.

(c) $u_{n}$ is a homogeneous polynomial in $P_{1}, \ldots, P_{n}$ of degree $n$, where $\operatorname{deg}\left(P_{j}\right)=j$, with rational coefficients.

(d) $u_{n}=-\frac{1}{2 n} P_{n}+$ terms involving $P_{1}, \ldots, P_{n-1}$.

Thus $\mathbb{Z}\left[P_{1}, \ldots, P_{n}\right] \subset \mathbb{Z}\left[u_{1}, \ldots, u_{n}\right]$ is a proper inclusion, but over $\mathbb{Q}$ they are the same.

At this point we have formulas for the action of the real Witt algebra on the coefficients of $\phi_{+}$. If we write

$$
\frac{1}{\phi_{-}\left(\frac{1}{w}\right)}=\frac{1}{\rho_{\infty}} w\left(1+\sum_{n \geq 1} l_{n} w^{n}\right)
$$

where $w=\frac{1}{z}$ is the standard coordinate at infinity, then we can also write down formulas for the action of the real Witt algebra on the coefficients of $\phi_{-}$. We will postpone this until the next section. 


\section{Reformulation of the variational formulas}

\subsection{Preliminary comments on representations}

Above we have considered a representation of the real Lie algebra $\overrightarrow{\mathcal{W}}$ by real derivations on a space of complex-valued functions on $\operatorname{Loop}^{1}(\mathbb{C} \backslash\{0\})$. This representation is real, in the sense that the set of real functions is stable, or equivalently that the action commutes with complex conjugation of functions.

To be precise, fix $\lambda \in \mathbb{C}$. The Duren-Schiffer formulas imply that there is a real representation of the real Lie algebra $\overrightarrow{\mathcal{W}}$ by real derivations on the spaces of complex-valued functions

$$
\mathbb{C}\left[u_{1}, \overline{u_{1}}, u_{2}, \ldots ; \rho_{0}, \rho_{0}^{-1}\right] \rho_{0}^{\lambda}, \quad \mathbb{C}\left[l_{1}, \overline{l_{1}}, l_{2}, \ldots ; \rho_{\infty}, \rho_{\infty}^{-1}\right] \rho_{\infty}^{-\lambda},
$$

and

$$
\mathbb{C}\left[u_{1}, \overline{u_{1}}, u_{2}, \ldots ; l_{1}, \overline{l_{1}}, l_{2}, \ldots ; \rho_{0}, \rho_{0}^{-1} ; \rho_{\infty}, \rho_{\infty}^{-1}\right] a^{\lambda} .
$$

Denote this real action of $\overrightarrow{\mathcal{W}}$ by $\pi_{0}$. By abstract nonsense there is an associated complex representation of $\mathcal{W}$ by complex derivations of the algebra of complex-valued functions of selfavoiding loops, defined by

$$
\pi(L)=\frac{1}{2}\left(\pi_{0}(\vec{L})-i \pi_{0}(\overrightarrow{i L})\right) .
$$

There is also a representation

$$
\bar{\pi}(\bar{L})=\frac{1}{2}\left(\pi_{0}(\vec{L})+i \pi_{0}(\overrightarrow{i L})\right) .
$$

This is a complex representation of $\overline{\mathcal{W}}=\mathcal{W}^{*}$ by complex derivations.

In turn, in terms of the real embedding (3.1)

$$
\pi_{0}(\vec{L})=\pi(L)+\bar{\pi}(\bar{L}) .
$$

The point of this translation is that the complex representations $\pi$ and $\bar{\pi}$ are easier to analyze. In fact (on proper domains) they can be expressed in terms of highest weight representations, and this allows us to access well-known results from the theory of highest weight representations of the Virasoro algebra (at the moment the central charge $c=0$, so that we are only considering the Witt algebra).

\subsection{Formulas for the representation $\pi$}

\section{Proposition 4.1.}

(a) $\left(\pi\left(L_{0}\right) \phi_{+}\right)(z)=\phi_{+}(z)-\frac{1}{2} z \phi_{+}^{\prime}(z)$. In particular

$$
\pi\left(L_{0}\right) \rho_{0}=\frac{1}{2} \rho_{0} \quad \text { and } \quad \pi\left(L_{0}\right) u_{k}=-\frac{1}{2} k u_{k}, \quad k \geq 1 .
$$

(b) For $n>0, \pi\left(L_{n}\right) \phi_{+}=\phi_{+}^{n+1}$. In particular

$$
\pi\left(L_{n}\right) \rho_{0}=0, \quad \pi\left(L_{n}\right) u_{k}=0 \quad k<n, \quad \pi\left(L_{n}\right) u_{n}=\rho_{0}^{n}
$$

and in general

$$
\pi\left(L_{n}\right) u_{k}=\rho_{0}^{n} p_{k-n}^{(n+1)}\left(u_{1}, u_{2}, \ldots\right),
$$


where

$$
\left(1+u_{1} z+u_{2} z^{2}+\cdots\right)^{n+1}=\sum_{l=0}^{\infty} p_{l}^{(n+1)} z^{l} .
$$

(c) $\pi\left(L_{-1}\right) \phi_{+}=1+u^{\prime}(z)\left(-1+u_{1} z\right)$. In particular

$$
\pi\left(L_{-1}\right) \rho_{0}=-u_{1}, \quad \pi\left(L_{-1}\right)\left(\rho_{0} u_{1}\right)=-3 u_{2}+2 u_{1}^{2}
$$

and in general

$$
\pi\left(L_{-1}\right)\left(\rho_{0} u_{n}\right)=-(n+2) u_{n+1}+(n+1) u_{n} u_{1} .
$$

Hence

$$
\pi\left(L_{-1}\right) u_{n}=\frac{n+2}{\rho_{0}}\left(u_{1} u_{n}-u_{n+1}\right) .
$$

(d) For $n>1, \pi\left(L_{-n}\right) \rho_{0}=\frac{1}{2} \rho_{0}^{-n+1} P_{n}\left(u_{1}, \ldots, u_{n}\right)$, where

$$
P_{n}\left(u_{1}, \ldots, u_{n}\right)=B_{0}(n)=\operatorname{Res}\left(\left(\frac{U^{\prime}(t)}{U(t)}\right)^{2} t^{-n+1}, t=0\right) .
$$

If $\operatorname{deg}\left(u_{j}\right)=j$, then $P_{n}$ is a homogeneous polynomial of degree $n$.

(e) For $k \geq 1$

$$
\pi\left(L_{-n}\right) u_{k}=\rho_{0}^{-n}\left(\frac{k}{2} u_{k} B_{0}(n)+\sum_{m=1}^{k}(k+1-m) u_{k-m} B_{-m}(n)\right) .
$$

Equivalently

$$
\pi\left(L_{-n}\right)\left(\rho_{0} u_{k}\right)=\rho_{0}^{-n+1} \sum_{m=0}^{k}(k+1-m) u_{k-m} B_{-m}(n)-\rho_{0}^{-n+1} \frac{k+1}{2} u_{k} B_{0}(n),
$$

where

$$
B_{m}(n)=\operatorname{Res}\left(\left(\frac{U^{\prime}(t)}{U(t)}\right)^{2} U(t)^{m} t^{-n+1}, t=0\right)=\operatorname{Res}\left(\frac{u(z)^{-n+1}}{u^{\prime}(z)} z^{m-2}, z=0\right) .
$$

Using Lemma 4.4 below, this can be restated in the following way.

\section{Proposition 4.2.}

(a) For $n \in \mathbb{Z}$

$$
\pi\left(L_{n}\right) \rho_{0}=\frac{1}{2} \rho_{0} \operatorname{Res}\left(\frac{\phi_{+}^{n+1}(z)}{z^{2} \phi_{+}^{\prime}(z)}, z=0\right) .
$$

(b) For $k \geq 1$

$$
\pi\left(L_{n}\right) u_{k}=\frac{k}{2} u_{k} \widetilde{B}_{0}(n)+\sum_{m=1}^{k}(k+1-m) u_{k-m} \widetilde{B}_{-m}(n) .
$$


Equivalently

$$
L_{n}\left(\rho_{0} u_{k}\right)=\rho_{0}^{n+1} \sum_{m=0}^{k}(k+1-m) u_{k-m} B_{-m}(n)-\rho_{0}^{n+1} \frac{k+1}{2} u_{k} B_{0}(n),
$$

where

$$
\widetilde{B}_{m}(n)=\operatorname{Res}\left(\frac{\phi_{+}(z)^{n+1}}{\phi_{+}^{\prime}(z)} z^{m-2}, z=0\right) .
$$

Remark 4.3. This second statement seems cleaner than the first. However, as we will see when we introduce the energy-momentum tensor, the first statement has the advantage of being stated in terms of the inverse of $\phi_{+}$.

To avoid cumbersome notation, we will often identify $L_{n}$ with its corresponding operator, $\pi\left(L_{n}\right)$. Suppose that we write $u_{0}=1$ and $a_{k}=\rho_{0} u_{k}$, so that

$$
\phi_{+}(z)=\sum_{k=0}^{\infty} a_{k} z^{k+1}
$$

If $n>0$ and $k \geq 1$, then according to (e)

$$
\begin{aligned}
L_{-n}\left(a_{k}\right)= & \sum_{m=0}^{k}(k+1-m) a_{k-m} \operatorname{Res}\left(\left(\frac{\left(\phi_{+}^{-1}\right)^{\prime}(t)}{\phi_{+}^{-1}(t)}\right)^{2} \phi_{+}^{-1}(t)^{-m} t^{-n+1}, t=0\right) \\
& -\frac{k+1}{2} a_{k} \operatorname{Res}\left(\left(\frac{\left(\phi_{+}^{-1}\right)^{\prime}(t)}{\phi_{+}^{-1}(t)}\right)^{2} t^{-n+1}, t=0\right)
\end{aligned}
$$

and

$$
\begin{aligned}
L_{-n}\left(\phi_{+}\right)= & \sum_{k=0}^{\infty}\left(\sum_{m=0}^{k}(k+1-m) a_{k-m} \operatorname{Res}\left(\left(\frac{\left(\phi_{+}^{-1}\right)^{\prime}(t)}{\phi_{+}^{-1}(t)}\right)^{2} \phi_{+}^{-1}(t)^{-m} t^{-n+1}, t=0\right)\right. \\
& \left.-\frac{1}{2}(k+1) a_{k} \operatorname{Res}\left(\left(\frac{\left(\phi_{+}^{-1}\right)^{\prime}(t)}{\phi_{+}^{-1}(t)}\right)^{2} t^{-n+1}, t=0\right)\right) z^{k+1} .
\end{aligned}
$$

\section{Lemma 4.4.}

$$
\operatorname{Res}\left(\left(\frac{\left(\phi_{+}^{-1}\right)^{\prime}(t)}{\phi_{+}^{-1}(t)}\right)^{2} \phi_{+}^{-1}(t)^{-m} t^{-n+1}, t=0\right)=\operatorname{Res}\left(\frac{\phi_{+}(z)^{-n+1}}{\phi_{+}^{\prime}(z) z^{2+m}}, z=0\right) .
$$

Proof. Fix a small circle $C$ surrounding 0 in the $t$ plane. Then

$$
\begin{aligned}
\int_{C}\left(\frac{\left(\phi_{+}^{-1}\right)^{\prime}(t)}{\phi_{+}^{-1}(t)}\right)^{2} \phi_{+}^{-1}(t)^{-m} t^{-n+1} d t & =\int_{\phi_{+}^{-1}(C)}\left(\phi_{+}^{-1}\right)^{\prime}\left(\phi_{+}(z)\right)^{2} \frac{\phi_{+}(z)^{-n+1}}{z^{m+2}} d \phi_{+}(z) \\
& =2 \pi i \operatorname{Res}\left(\frac{\phi_{+}(z)^{-n+1}}{\phi_{+}^{\prime}(z) z^{2+m}}, z=0\right) .
\end{aligned}
$$

This can be restated more cleanly in the following way. 


\section{Lemma 4.5.}

$$
\rho_{0}^{-n} B_{m}(n)=\operatorname{Res}\left(\frac{\phi_{+}^{-n+1}(z)}{\phi_{+}^{\prime}(z)} z^{m-2}, z=0\right) .
$$

Using the lemma we can write

$$
\begin{aligned}
L_{-n}\left(\phi_{+}\right)= & \sum_{k=0}^{\infty}\left(\sum_{m=0}^{k}(k+1-m) a_{k-m} \operatorname{Res}\left(\frac{\phi_{+}(s)^{-n+1}}{\phi_{+}^{\prime}(s) s^{2+m}}, s=0\right)\right. \\
& \left.-\frac{1}{2}(k+1) a_{k} \operatorname{Res}\left(\frac{\phi_{+}(s)^{-n+1}}{\phi_{+}^{\prime}(s) s^{2}}, s=0\right)\right) z^{k+1} \\
= & \phi_{+}^{\prime}(z)\left(\sum_{m=0}^{\infty}\left(\frac{\phi_{+}^{-n+1}}{\phi_{+}^{\prime}}\right)_{m+1} z^{m+1}-z \operatorname{Res}\left(\frac{\phi_{+}(s)^{-n+1}}{\phi_{+}^{\prime}(s) s^{2}}, s=0\right)\right) \\
= & \phi_{+}^{\prime}(z)\left(\left(\frac{\phi_{+}(z)^{-n+1}}{\phi_{+}^{\prime}(z)}\right)_{++}-z \operatorname{Res}\left(\frac{\phi_{+}(s)^{-n+1}}{\phi_{+}^{\prime}(s) s^{2}}, s=0\right)\right) .
\end{aligned}
$$

The pleasant surprise is that this expression leads to a formula which is valid for all $n$.

Theorem 4.6. For any $n \in \mathbb{Z}$

$$
L_{n}\left(\phi_{+}\right)(z)=\phi_{+}^{\prime}(z)\left(\frac{\phi_{+}(z)^{n+1}}{\phi_{+}^{\prime}(z)}\right)_{++}-\frac{1}{2} z \phi_{+}^{\prime}(z) \operatorname{Res}\left(\frac{\phi_{+}(s)^{n+1}}{\phi_{+}^{\prime}(s) s^{2}}, s=0\right)
$$

and

$$
L_{n}(u)(z)=\rho_{0}^{n}\left(u^{\prime}(z)\left(\frac{u(z)^{n+1}}{u^{\prime}(z)}\right)_{++}-\frac{1}{2}\left(z u^{\prime}(z)+u(z)\right) \operatorname{Res}\left(\frac{u(s)^{n+1}}{u^{\prime}(s) s^{2}}, s=0\right)\right) .
$$

Proof. We just need to check that this formula agrees with our previous calculations when $n \geq 0$. This is straightforward.

\subsection{Formulas for $\bar{\pi}$}

\section{Proposition 4.7.}

(a) $\bar{\pi}\left(\bar{L}_{0}\right) \phi_{+}=\frac{1}{2} z \phi_{+}^{\prime}(z)$. In particular

$$
\bar{\pi}\left(\bar{L}_{0}\right) \rho_{0}=\frac{1}{2} \rho_{0} \quad \text { and } \quad \bar{\pi}\left(\bar{L}_{0}\right) u_{k}=\frac{k-1}{2} u_{k}, \quad k \geq 1 .
$$

(b) For $n>0, \bar{\pi}\left(\bar{L}_{n}\right) \phi_{+}=0$.

(c) $\bar{\pi}\left(\bar{L}_{-1}\right) \phi_{+}=u^{\prime}(z)\left(-u_{1}^{*} z+z^{2}\right)$. In particular

$$
\bar{\pi}\left(\bar{L}_{-1}\right) \rho_{0}=-u_{1}^{*}, \quad \bar{\pi}\left(\bar{L}_{-1}\right) u_{1}=\rho_{0}^{-1}\left(1-u_{1} u_{1}^{*}\right) .
$$

In general

$$
\bar{\pi}\left(\bar{L}_{-1}\right) u_{n}=\rho_{0}^{-1} n\left(u_{n-1}-u_{1}^{*} u_{n}\right) .
$$

(d) For $n>1, \bar{\pi}\left(\bar{L}_{-n}\right) \rho_{0}=\frac{1}{2} \rho_{0}^{-n+1} P_{n}\left(u_{1}, \ldots, u_{n}\right)^{*}$. 
(e) For $k \geq 1$

$$
\bar{L}_{-n} u_{k}=\rho_{0}^{-n}\left(\frac{k}{2} u_{k} B_{0}(n)^{*}+\sum_{m=1}^{k}(k+1-m) u_{k-m} B_{m}(n)^{*}\right) .
$$

Equivalently

$$
\bar{L}_{-n}\left(\rho_{0} u_{k}\right)=\rho_{0}^{-n+1} \sum_{m=0}^{k}(k+1-m) u_{k-m} B_{m}(n)^{*}-\rho_{0}^{-n+1} \frac{k+1}{2} u_{k} B_{0}(n)^{*} .
$$

Now we want to add things up as in the preceding section. As before we write $\phi_{+}(z)=$ $\sum a_{k} z^{k+1}$, where $a_{k}=\rho_{0} u_{k}$ and it is understood that $u_{0}=1$. By part (e)

$$
\bar{L}_{-n}\left(a_{k}\right)=\rho_{0}^{-n} \sum_{m=0}^{k}(k+1-m) a_{k-m} B_{m}(n)^{*}-\rho_{0}^{-n} \frac{k+1}{2} a_{k} B_{0}(n)^{*}
$$

By the change of variable lemma of the preceding subsection

$$
\rho_{0}^{-n} B_{m}(n)=\operatorname{Res}\left(\frac{\phi_{+}(z)^{-n+1}}{\phi_{+}^{\prime}(z)} z^{m-2}, z=0\right) .
$$

Therefore

$$
\bar{L}_{-n} \phi_{+}(z)=\phi_{+}^{\prime}(z) \sum_{m=0}^{\infty}\left(\left(\frac{\phi_{+}(z)^{-n+1}}{\phi_{+}^{\prime}(z)}\right)_{-m+1}\right)^{*} z^{m+1}-\frac{1}{2} z \phi_{+}^{\prime}(z)\left(\left(\frac{\phi_{+}(z)^{-n+1}}{\phi_{+}^{\prime}(z)}\right)_{1}\right)^{*},
$$

where the notation $(\cdots)_{k}$ denotes the $k$ th Fourier coefficient. This equals

$$
\begin{gathered}
\phi_{+}^{\prime}(z) \sum_{m=0}^{\infty}\left[\left(\frac{\phi_{+}(z)^{-n+1}}{\phi_{+}^{\prime}(z)}\right)^{*} z^{2}\right]_{m+1} z^{m+1}-\frac{1}{2} z \phi_{+}^{\prime}(z)\left(\left(\frac{\phi_{+}(z)^{-n+1}}{\phi_{+}^{\prime}(z)}\right)_{1}\right)^{*} \\
=\left[\left(z^{-2} \frac{\phi_{+}^{-n+1}(z)}{\phi_{+}^{\prime}(z)}\right)^{*}\right]_{++}-\frac{1}{2} z \phi_{+}^{\prime}(z)\left(\left(\frac{\phi_{+}(z)^{-n+1}}{\phi_{+}^{\prime}(z)}\right)_{1}\right)^{*} .
\end{gathered}
$$

As in the preceding subsection, we obtain the following uniform formula.

Theorem 4.8. For any $n \in \mathbb{Z}$

$$
\bar{L}_{n} \phi_{+}(z)=\phi_{+}^{\prime}(z)\left(\left[\left(z^{-2} \frac{\phi_{+}^{n+1}(z)}{\phi_{+}^{\prime}(z)}\right)^{*}\right]_{++}-\frac{1}{2} z\left(\left(\frac{\phi_{+}(z)^{n+1}}{\phi_{+}^{\prime}(z)}\right)_{1}\right)^{*}\right) .
$$

Proof. We just need to check that this formula agrees with the formulas in Proposition 4.7. This is again straightforward.

\subsection{Formulas for $\pi_{0}$, revisited}

We can use Theorems 4.6 and 4.8 to recast the Duren-Schiffer variational formulas in the following form. 
Corollary 4.9. For all $n \in \mathbb{Z}, \vec{L}_{n} \phi_{+}$equals

$$
\begin{aligned}
\phi_{+}^{\prime}(z) & {\left[\frac{\phi_{+}(z)^{n+1}}{\phi_{+}^{\prime}(z)}+\left(z^{-2} \frac{\phi_{+}(z)^{n+1}}{\phi_{+}^{\prime}(z)}\right)^{*}\right]_{++} } \\
& -\frac{1}{2} z\left(\operatorname{Res}\left(\frac{\phi_{+}(s)^{n+1}}{\phi_{+}^{\prime}(s) s^{2}}, s=0\right)+\operatorname{Res}\left(\frac{\phi_{+}(s)^{n+1}}{\phi_{+}^{\prime}(s) s^{2}}, s=0\right)^{*}\right) \\
= & \phi_{+}^{\prime}(z)\left(\frac{1}{2}\left(c_{1}+\overline{c_{1}}\right) z+\sum_{k>1}\left(c_{k}+\overline{c_{2-k}}\right) z^{k}\right),
\end{aligned}
$$

where

$$
\frac{\phi_{+}(z)^{n+1}}{\phi_{+}^{\prime}(z)}=\sum_{k=n+1}^{+\infty} c_{k} z^{k} .
$$

Proof. By definition

$$
\vec{L}_{n} \phi_{+}=L_{n} \phi_{+}+\bar{L}_{n} \phi_{+} .
$$

Theorems 4.6 and 4.8 imply that this equals

$$
\begin{aligned}
\phi_{+}^{\prime}(z) & {\left[\frac{\phi_{+}(z)^{n+1}}{\phi_{+}^{\prime}(z)}\right]_{++}-\frac{1}{2} z \phi_{+}^{\prime}(z) \operatorname{Res}\left(\frac{\phi_{+}(s)^{n+1}}{\phi_{+}^{\prime}(s) s^{2}}, s=0\right) } \\
+ & \phi_{+}^{\prime}(z)\left[\left(z^{-2} \frac{\phi_{+}(z)^{n+1}}{\phi_{+}^{\prime}(z)}\right)^{*}\right]_{++}-\frac{1}{2} z \phi_{+}^{\prime}(z)\left(\left(\frac{\phi_{+}(z)^{n+1}}{\phi_{+}^{\prime}(z)}\right)_{1}\right)^{*} .
\end{aligned}
$$

It is obviously desirable to find a direct proof of these formulas which reflects their structure.

\subsection{Calculations with $\phi_{-}$}

On the one hand, in the standard $w$ coordinate at $\infty \in \mathbb{P}^{1}$,

$$
\frac{1}{\phi_{-}\left(\frac{1}{w}\right)}=\frac{1}{\rho_{\infty}} w\left(1+\sum_{n=1}^{\infty} l_{n} w_{n}\right) .
$$

The $l_{n}$ coordinates for $\phi_{-}$are analogous to the $u_{n}$ coordinates for $\phi_{+}$, and variational formulas for $\phi_{-}$essentially arise from substituting $l_{j}$ 's for $u_{j}$ 's in our earlier formulas. On the other hand, in the standard $z$ coordinate,

$$
\phi_{-}(z)=\rho_{\infty} L(z)=\rho_{\infty} z\left(1+\sum_{m=1}^{\infty} b_{m} z^{-m}\right)
$$

and it is occasionally useful to employ the $b_{m}$ coordinates. The relation between the two sets of coordinates is standard.

\section{Lemma 4.10.}

$$
\mathbb{C}\left[l_{1}, l_{2}, \ldots\right]=\mathbb{C}\left[b_{1}, b_{2}, \ldots\right] .
$$

In fact for each $M$

$$
\mathbb{C}\left[l_{1}, l_{2}, \ldots, l_{M}\right]=\mathbb{C}\left[b_{1}, b_{2}, \ldots, b_{M}\right] .
$$




\section{Proof.}

$$
w\left(1+\sum_{n=1}^{\infty} l_{n} w^{n}\right)=\frac{1}{\frac{1}{w}\left(1+\sum_{m=1}^{\infty} b_{m} w^{m}\right)}
$$

or

$$
1+\sum_{n=1}^{\infty} l_{n} w^{n}=\frac{1}{1+\sum_{m=1}^{\infty} b_{m} w^{m}}
$$

implies

$$
l_{1}=-b_{1}, \quad l_{2}=-b_{2}+b_{1}^{2}, \quad \ldots .
$$

The $\phi_{-}$analog of Theorems 4.6 and 4.8 is the following theorem. In the statement, for a Laurent expansion convergent in an annulus $R<|z|<\infty$, we use the notation $\operatorname{Res}\left(\sum g_{m} z^{m}, z=\right.$ $\infty)=-g_{-1}$ (This is actually the residue of the differential $g(z) d z$ at $z=\infty$ in the Riemann sphere).

Theorem 4.11. Let $n \in \mathbb{Z}$.

(a)

$$
L_{n}\left(\phi_{-}(z)\right)=-z^{2} \phi_{-}^{\prime}(z)\left[\left(\frac{\phi_{-}(z)^{n+1}}{z^{2} \phi_{-}^{\prime}(z)}\right)_{-}+\frac{z^{-1}}{2} \operatorname{Res}\left(\frac{\phi_{-}(t)^{n+1}}{t^{2} \phi_{-}^{\prime}(t)}, t=\infty\right)\right]
$$

and

$$
\begin{aligned}
L_{n}(L(z))= & -\frac{\rho_{\infty}^{n}}{2} \operatorname{Res}\left(\frac{L(t)^{n+1}}{t^{2} L^{\prime}(t)}, t=\infty\right) L(z) \\
& -\rho_{\infty}^{n} z^{2} L^{\prime}(z)\left[\left(\frac{L(z)^{n+1}}{z^{2} L^{\prime}(z)}\right)_{-}+\frac{z^{-1}}{2} \operatorname{Res}\left(\frac{L(t)^{n+1}}{t^{2} L^{\prime}(t)}, t=\infty\right)\right] .
\end{aligned}
$$

(b)

$$
\bar{L}_{n}\left(\phi_{-}(z)\right)=-z^{2} \phi_{-}^{\prime}(z)\left[\frac{z^{-1}}{2} \operatorname{Res}\left(\frac{\phi_{-}(t)^{n+1}}{t^{2} \phi_{-}^{\prime}(t)}, \infty\right)^{*}+\left(\left(\frac{\phi_{-}(z)^{n+1}}{\phi_{-}^{\prime}(z)}\right)^{*}\right)_{-}\right]
$$

and

$$
\begin{aligned}
\bar{L}_{n}(L(z))= & -\frac{\rho_{\infty}^{n}}{2}\left(L(z)+z L^{\prime}(z)\right) \operatorname{Res}\left(\frac{L(t)^{n+1}}{t^{2} L^{\prime}(t)}, t=\infty\right)^{*} \\
& -\rho_{\infty}^{n} z^{2} L^{\prime}(z)\left(\left(\frac{L(z)^{n+1}}{L^{\prime}(z)}\right)^{*}\right)_{-} .
\end{aligned}
$$

\subsection{Representation-theoretic consequences}

The formulas of the preceding section imply that $\pi$ is a complex representation of the Witt algebra $\mathcal{W}$ by derivations of the algebra $\Omega^{0}\left(\rho_{0}\right) \otimes \mathbb{C}\left[u_{1}, u_{2}, \ldots\right]$, where $\Omega^{0}\left(\rho_{0}\right)$ denotes any algebra of smooth functions of $\rho_{0}$.

Consider the action of $\mathcal{W}$ on the vector space

$$
\mathbb{C}\left[\rho_{0}^{\lambda}, \rho_{0}, \rho_{0}^{-1}, u_{1}, u_{2}, \ldots\right],
$$


where $\lambda$ is a fixed complex number. For $n>0$ the operators $L_{n}$ kill $\rho_{0}^{\lambda}$, and the spectrum of $L_{0}$ on the $\mathcal{W}$-module generated by $\rho_{0}^{\lambda}$ is $\{\lambda / 2+n: n=0,1, \ldots\}$. We will refer to this as a lowest weight module (admittedly there are conflicting conventions). The following proposition follows from well-known facts about such representations (see [11]).

Proposition 4.12. For any $\lambda \in \mathbb{C}$,

(a) The representation generated by the $\pi$ action of $\mathcal{W}$ on $\rho_{0}^{\lambda}$ is a realization of the unique irreducible lowest weight representation of the Virasoro algebra with central charge $c=0$ and $h=\frac{1}{2} \lambda$. If $\lambda \neq-\frac{m^{2}-1}{12}$, then

$$
\pi(\mathcal{U}(\mathcal{W})) \rho_{0}^{\lambda}=\bigoplus_{n=0}^{\infty} \rho_{0}^{\lambda-n} \mathbb{C}\left[u_{1}, u_{2}, \ldots\right]^{(n)},
$$

where $u_{j}$ has degree $j$. Otherwise there is a proper containment.

(b) Similarly, the representation generated by the $\pi$ action of $\mathcal{W}$ on $\rho_{\infty}^{-\lambda}$ is a realization of the highest weight representation of the Virasoro algebra with central charge $c=0$ and $h=-\frac{\lambda}{2}$. If $\lambda \neq-\frac{m^{2}-1}{12}$, then

$$
\pi(\mathcal{U}(\mathcal{W})) \rho_{\infty}^{-\lambda}=\bigoplus_{n=0}^{\infty} \rho_{\infty}^{-\lambda-n} \mathbb{C}\left[l_{1}, l_{2}, \ldots\right]^{(n)}
$$

where $l_{j}$ has degree $j$. Otherwise there is a proper containment.

Remark 4.13. The realization of the lowest weight representation in part (a) is related in a relatively simple way to the realization, using geometric quantization techniques, due to Kirillov and Yuriev in [12]. In [12] $\mathcal{W}$ acts on a space of sections of a line bundle (parameterized by $c=0$ and $h=\lambda / 2$ ) over (a somewhat imprecisely defined) space of Schlicht functions $u \in \mathcal{S}$ (normalized univalent functions on the disk, viewed as a homogeneous space for $\operatorname{Diff}\left(S^{1}\right)$ ). In coordinates (by trivializing the line bundle) this vector space is identified with $\mathbb{C}\left[u_{1}, u_{2}, \ldots\right]$, polynomials in the coefficients of the univalent function $u$, and the formulas for the action appear in (8) of [12] (with $c=0$, and one takes the negative of the operators, because we consider the opposite of the bracket in [12]). The intertwining operator from Kirillov and Yuriev's realization to our realization in (a) is given by the map

$$
\mathbb{C}\left[u_{1}, u_{2}, \ldots\right] \rightarrow \mathbb{C}\left[\rho_{0}^{\lambda}, \rho_{0}, \rho_{0}^{-1}, u_{1}, u_{2}, \ldots\right]: P\left(u_{1}, u_{2}, \ldots\right) \mapsto P\left(U_{1} / \rho_{0}, U_{2} / \rho_{0}^{2}, \ldots\right) \rho_{0}^{\lambda},
$$

where $U=t\left(1+\sum_{n>0} U_{n} t^{n}\right)$ is the inverse to the univalent function $u=z\left(1+\sum_{n>0} u_{n} z^{n}\right)$. An advantage of our realization is that the operators are derivations of an algebra, which makes them more amenable to calculations. This will appear in the first author's dissertation.

\subsection{Stress-energy formulation}

Consider the standard holomorphic coordinate $z=x+i y$. In real coordinates the symmetric stress tensor has the form

$$
\mathcal{T}=\left(\begin{array}{ll}
d x & d y
\end{array}\right)\left(\begin{array}{ll}
T_{11} & T_{12} \\
T_{21} & T_{22}
\end{array}\right)\left(\begin{array}{l}
d x \\
d y
\end{array}\right)
$$

where $T_{12}=T_{21}$. In complex coordinates

$$
T=\left(\begin{array}{ll}
d z & d \bar{z}
\end{array}\right)\left(\begin{array}{cc}
T_{11}-T_{22}-2 i T_{12} & T_{11}+T_{22}+i\left(T_{12}-T_{21}\right) \\
T_{11}-T_{22}+i\left(T_{21}-T_{12}\right) & T_{11}-T_{22}+2 i T_{12}
\end{array}\right)\left(\begin{array}{l}
d z \\
d \bar{z}
\end{array}\right)
$$




$$
=\left(\begin{array}{ll}
d z & d \bar{z}
\end{array}\right)\left(\begin{array}{cc}
T_{11}-T_{22}-2 i T_{12} & T_{11}+T_{22} \\
T_{11}+T_{22} & T_{11}+T_{22}+2 i T_{12}
\end{array}\right)\left(\begin{array}{l}
d z \\
d \bar{z}
\end{array}\right)
$$

Conformal invariance is implied by the trace condition

$$
\operatorname{tr}(T)=T_{11}+T_{22}=0
$$

(see [7, p. 101 and p. 103]). In complex coordinates this implies that $T$ is diagonal.

In a conformal field theory with central charge $c=0$

$$
T(z):=\left(T_{11}-T_{22}-2 i T_{12}\right) d z^{2}=\sum_{n=-\infty}^{\infty} L_{n} z^{-n}\left(\frac{d z}{z}\right)^{2}
$$

is a holomorphic quadratic differential (see [7, p. 155]; note: for $c \neq 0$, the stress energy "tensor" is actually a holomorphic projective connection; see [7, p. 136] or [15, p. 532]).

We are seeking a completely natural formulation for the action of the Witt algebra

\section{Proposition 4.14.}

$$
T(t) \rho_{0}=\frac{\rho_{0}}{2}\left(\partial \log \left(\phi_{+}^{-1}(t)\right)\right)^{2}, \quad T(t) \rho_{\infty}=-\frac{\rho_{\infty}}{2}\left(\partial \log \left(\phi_{-}^{-1}(t)\right)\right)^{2} .
$$

Proof. By definition

$$
T(t) \rho_{0}=\sum_{n=-\infty}^{\infty} L_{-n}\left(\rho_{0}\right) t^{n}\left(\frac{d t}{t}\right)^{2} .
$$

By part (a) of Proposition 4.2, this equals

$$
\frac{1}{2} \rho_{0} \sum_{n=0}^{\infty} \operatorname{Res}\left(\left(\frac{\left(\phi_{+}^{-1}\right)^{\prime}(t)}{\phi_{+}^{-1}(t)}\right)^{2} t^{-n+1}, t=0\right) t^{n}\left(\frac{d t}{t}\right)^{2}=\frac{\rho_{0}}{2}\left(\partial \log \left(\phi_{+}^{-1}(t)\right)\right)^{2} .
$$

This proves the first statement. The proof of the second statement is similar.

\section{Corollary 4.15. In the sense of hyperfunctions}

$$
T(t) a^{\lambda}=\frac{\lambda}{2}\left(\left(\partial \log \left(\phi_{+}^{-1}(t)\right)\right)^{2}+\left(\partial \log \left(\phi_{-}^{-1}(t)\right)\right)^{2}\right) a^{\lambda} .
$$

Proof. From a formal power series point of view, this follows immediately from the proposition. From the point of view of analysis, this equality has to be interpreted in a hyperfunction sense, because the first term is holomorphic in $U_{+}$and the second term is holomorphic in $U_{-}$.

\section{Infinitesimal invariance}

Suppose that $\gamma \in \operatorname{Loop}^{1}(\mathbb{C} \backslash\{0\})$. In terms of the standard coordinate $z$,

$$
\phi_{+}(z)=\rho_{0}(\gamma) u(z), \quad u(z)=z\left(1+\sum_{n \geq 1} u_{n} z^{n}\right) .
$$

In terms of the coordinate $w=\frac{1}{z}$,

$$
\frac{1}{\phi_{-}\left(\frac{1}{w}\right)}=\frac{1}{\rho_{\infty}} w\left(1+\sum_{n \geq 1} l_{n} w^{n}\right)
$$


The variational formulas of the preceding section imply that the vector space of functions of the form

$$
p\left(u_{1}, \ldots, u_{n}, \overline{u_{1}}, \ldots, \overline{u_{n}}\right) f\left(\rho_{0}\right),
$$

where $p$ is a polynomial of any number of variables, and $f$ has compact support in $\mathbb{R}^{+}$, is stable with respect to the action of the Witt algebra (this applies both to the real action and the complexified actions). Since the Witt algebra is stable with respect to $z \mapsto w=\frac{1}{z}$, the vector space of functions of the form

$$
p\left(l_{1}, \ldots, l_{n}, \overline{l_{1}}, \ldots, \overline{l_{n}}\right) f\left(\rho_{\infty}\right),
$$

where $p$ is a polynomial of any number of variables, and $f$ has compact support in $\mathbb{R}^{+}$, is also stable with respect to the action of the Witt algebra. Consequently the vector space of "test functions" spanned by functions of the form

$$
F=p\left(u_{1}, \ldots, \overline{u_{1}}, \ldots, l_{1}, \ldots, \overline{l_{1}}, \ldots\right) f\left(\rho_{0}, \rho_{\infty}\right)
$$

where $p$ is a polynomial and $f$ has compact support in $\mathbb{R}^{+} \times \mathbb{R}^{+}$, is stable with respect to the Witt algebra (for the real or complexified actions). In reference to $F$, since $u_{n}$ and $l_{n}$ are bounded (by constants depending only on $n$ ), $p$ is bounded. The compact support condition on $f$ implies that $F$ is supported on Loop ${ }^{1}$ of a fixed finite type annulus. Since $\mu_{0}$ has finite measure on loops in a finite type annulus, $F$ is integrable.

Proposition 5.1. The measure $\mu_{0}$ is infinitesimally conformally invariant, in the sense that for any $L \in \mathcal{W} \times \overline{\mathcal{W}}$

$$
\int L(F) d \mu_{0}(\gamma)=0
$$

for any test function $F$ as in (5.1).

Proof. It suffices to prove the proposition for $\vec{L} \in \overrightarrow{\mathcal{W}}$

By Koebe's theorem, a test function $F$ as in (5.1) is supported on $\operatorname{Loop}^{1}\left(\left\{\delta<|z|<\delta^{-1}\right\}\right)$ for some $\delta$. Let $A_{0}$ denote a finite type annulus containing $\left\{\delta \leq|z| \leq \delta^{-1}\right\}$. For some positive $t_{0}$, for all $|t|<t_{0}$, the flow $\exp (t \vec{L})$ is defined on $A_{0}$, and $\left\{\delta \leq|z| \leq \delta^{-1}\right\}$ will be contained in $\cap_{t<t_{0}} A_{t}$, where $A_{t}:=\exp (t \vec{L}) A_{0}$. By local conformal invariance

$$
\int F d \mu_{0}=\int F d \mu_{A_{0}}=\int\left(e^{t \vec{L}}\right)_{*} F d \mu_{A_{t}}=\int\left(e^{t \vec{L}}\right)_{*} F d \mu_{0}
$$

To complete the proof we need to justify taking the derivative with respect to $t$ at $t=0$ under the last integral. The derivative $\vec{L} F$ is another test function, necessarily bounded. The translates

$$
\left(e^{t \vec{L}}\right)_{*}(\vec{L} F), \quad|t|<t_{0}
$$

are also uniformly bounded by the same constant. Moreover the translates (5.2) are all supported on Loop ${ }^{1}$ of some finite type annulus, for which the $\mu_{0}$ measure is finite. Thus a multiple of the characteristic function of Loop ${ }^{1}$ for this fixed finite type annulus is integrable and dominates all of the translates (5.2). Hence by dominated convergence we can differentiate under the integral sign. 
Kontsevich and Suhov have conjectured that there is a converse of this result which holds generally for their conjectural family of measures $\mu_{c}$ deforming $\mu_{0}$ (see Section 2.5.2 of [13]).

For the purposes of this paper, we need to be able to apply integration by parts to functions which involve the bounded function $a^{\lambda}(\lambda>0)$, rather than a function having compact support in $\rho_{0}, \rho_{\infty}$. One complication is that for $\vec{L} \in \overrightarrow{\mathcal{W}}$,

$$
\vec{L} a^{\lambda}=\lambda a^{\lambda-1} \vec{L}(a)
$$

is not necessarily bounded.

Lemma 5.2. Suppose that

$$
F=p(u, \bar{u}, l, \bar{l}) f\left(\rho_{\infty}\right) a^{\lambda},
$$

where $p$ is a polynomial and $f$ has compact support in $\mathbb{R}^{+}$. Then for any $L \in \mathcal{W} \times \overline{\mathcal{W}}$, for $\operatorname{Re}(\lambda)$ sufficiently large,

$$
\int L(F) d \mu_{0}(\gamma)=0
$$

The same conclusion applies if we replace $f\left(\rho_{\infty}\right)$ by $f\left(\rho_{0}\right)$.

Proof. Fix a smooth positive function $g\left(\rho_{0}\right)$ having compact support for $\rho_{0} \in \mathbb{R}^{+}$and identically 1 in a neighborhood of $\rho=1$. By Proposition 5.1, for each $\delta>0$,

$$
\int L\left(g\left(\delta \rho_{0}\right) F\right) d \mu_{0}(\gamma)=0
$$

or

$$
\delta \int g^{\prime}\left(\delta \rho_{0}\right) L\left(\rho_{0}\right) F d \mu_{0}+\int g\left(\delta \rho_{0}\right) L(F) d \mu_{0}(\gamma)=0
$$

Since $g$ is fixed, the first term goes to zero as $\delta \rightarrow 0$. We can apply dominated convergence to the second term, for sufficiently large $\lambda$ (so that the part of the integrand not involving $g$ is bounded, and hence the integral is well-defined). This implies the Lemma.

Proposition 5.3. Suppose that $F=a^{\lambda} p(u, \bar{u}, l, \bar{l})$, where $p$ is a polynomial.

(a) If $L=L_{n}$ or $\bar{L}_{n}$ with $n \leq 0$, then for sufficiently large $\operatorname{Re}(\lambda)$

$$
\left.\int L(F)\right|_{\rho_{\infty}=1} d \nu_{0}=0 \text {. }
$$

(b) If $L=L_{n}$ or $\bar{L}_{n}$ with $n \geq 0$, then for sufficiently large $\operatorname{Re}(\lambda)$

$$
\left.\int L(F)\right|_{\rho_{0}=1} d \nu_{0}=0 \text {. }
$$

Proof. Suppose that $L=L_{n}$ or $\bar{L}_{n}$ with $n<0$. Fix a smooth family of functions $g_{\delta}\left(\rho_{\infty}\right)$ which converges to the $\delta$ function at $\rho_{\infty}=1$. Using $L_{n}\left(\rho_{\infty}\right)=0$ and Lemma 5.2,

$$
\int L\left(F g_{\delta}\left(\rho_{\infty}\right)\right) d \mu_{0}=\int L(F) g_{\delta}\left(\rho_{\infty}\right) d \mu_{0}=0 .
$$

Since $L(F)$ is bounded for sufficiently large $\operatorname{Re}(\lambda)$, the left hand side of the last equality converges to $\int L(F) d \nu_{0}$ as $\delta \rightarrow 0$. This implies part (a).

If $L=L_{n}$ or $\bar{L}_{n}$ with $n>0$, the same argument applies with $g_{\delta}\left(\rho_{0}\right)$ in place of $g_{\delta}\left(\rho_{\infty}\right)$.

If $L=\vec{L}_{0}$, then $L(F)=0$. We have previously observed that if $L=\overrightarrow{i L}_{0}$, then $L$ exponentiates to rotational symmetry of $\mathbb{C} \backslash\{0\}$, and this corresponds to invariance of $\nu_{0}$ with respect to the conjugation action of rotations on homeomorphisms. 
In the sections below, we will repeatedly apply a variation of the preceding proof in the following way. Suppose that $n>0$ and $L=L_{n}$ or $L=\bar{L}_{n}$. Then as in the proof

$$
\int L\left(\rho^{-n} F g_{\delta}\left(\rho_{0}\right)\right) d \mu_{0}=\int L(F) \rho_{0}^{-n} g_{\delta}\left(\rho_{0}\right) d \mu_{0}=0
$$

We can take the limit as $\delta \rightarrow 0$, because the support of $g_{\delta}$ remains bounded, and $\rho_{0}^{-n}$ will be bounded in this support region. This implies

$$
\int L(F) \rho_{0}^{-n} d \nu_{0}=0
$$

which can be written heuristically as

$$
\int L(F) \rho_{0}^{-n} \delta_{1}\left(\rho_{0}\right) d \mu_{0}=0
$$

where $\delta_{1}$ denotes the Dirac delta function at 1 . There are similar integral formulas involving $L_{-n}$, but then we must use an approximation to $\delta_{1}\left(\rho_{\infty}\right)$.

\section{Calculating moments}

Throughout this section, to simplify notation, we will write $E(\cdot)=\int(\cdot) d \nu_{0}$.

\subsection{The basic idea}

Suppose that $n>0$. The basic observation is that if $p(u)$ is homogeneous of degree $n$, where $\operatorname{deg}\left(u_{j}\right)=j$, then $\bar{L}_{-n}\left(\rho_{0}^{n} p(u)\right)$ does not depend upon $\rho_{0}$. Recall also that $\bar{L}_{-n}\left(\rho_{\infty}\right)=0$. We can now apply infinitesimal invariance to obtain

$$
E\left(\bar{L}_{-n}\left(\rho_{0}^{n} p(u)\right)\right)=\int \bar{L}_{-n}\left(\rho_{0}^{n} p(u)\right) \delta_{1}\left(\rho_{\infty}\right) d \mu_{0}=0,
$$

which gives rise to an integral formula.

To prove Theorem 1.7, we use the identity

$$
\bar{L}_{-1}\left(\rho_{0} u_{n+1} \bar{u}_{n}\right)=-(n+2) u_{n+1} \bar{u}_{n+1}+(n+1) u_{n} \bar{u}_{n} .
$$

\section{Theorem 6.1.}

$$
\int u_{n} u_{n}^{*} d \nu_{0}=\frac{1}{n+1}
$$

Proof. Formula (6.1), together with infinitesimal invariance, implies the recursion relation

$$
-(n+2) E\left(u_{n+1} \bar{u}_{n+1}\right)+(n+1) E\left(u_{n} \bar{u}_{n}\right)=0
$$

with the initial condition $E\left(u_{0} \bar{u}_{0}\right)=E(1)=1$.

We will use the following notation throughout this section.

\section{Definition 6.2.}

(a)

$$
\mathbb{C}[u]^{(n)}:=\operatorname{span}\left\{\prod_{k \geq 1} u_{k}^{p_{k}}: \sum_{k \geq 1} k p_{k}=n\right\},
$$

i.e. the $e^{i n}$ eigenspace for the action of rotations; $\mathbb{C}[\bar{u}]^{(n)}$ is defined similarly. 
(b) For each $n \geq 1$ we denote

$$
\mathbb{C}[u, \bar{u}]^{(n, n)}:=\operatorname{span}\left\{\prod_{k \geq 1} u_{k}^{p_{k}} \bar{u}_{k}^{q_{k}}: \sum_{k \geq 1} k p_{k}=\sum_{k \geq 1} k q_{k}=n\right\}
$$

or in other words $\mathbb{C}[u, \bar{u}]^{(n, n)} \cong \mathbb{C}[u]^{(n)} \otimes \mathbb{C}[\bar{u}]^{(n)}$. We will refer to elements in the vector space $\mathbb{C}[u, \bar{u}]^{(n, n)}$ as being of level $n$.

Note that the dimension of $\mathbb{C}[u]^{(n)}$ is $p(n)$, the number of partitions of $n$, hence grows very rapidly.

The rationale for the notation is the following. The outer tensor product, $\mathcal{W} \times \overline{\mathcal{W}}$, acts on the tensor product $\mathbb{C}[u] \otimes \mathbb{C}[\bar{u}]$. The product of the corresponding rotation groups acts, and induces a bigrading. In (b) we are considering the 0-eigenspace for the real embedded rotation group.

If $x \in \mathbb{C}[u, \bar{u}]^{\left(n, n^{\prime}\right)}$, then one may verify

$$
E(x)=e^{i\left(n-n^{\prime}\right)} E(x)
$$

using the rotational invariance of Werner's measure. Therefore, we restrict ourselves to computing integrals of elements at levels $n=1,2, \ldots$ (i.e., $n=n^{\prime}$ ).

Suppose $1 \leq m \leq n$. In general, we can obtain integral identities by computing

$$
\bar{L}_{-m} \circ \rho_{0}^{m}: \mathbb{C}[u, \bar{u}]^{(n, n-m)} \longrightarrow \bigoplus_{j=0}^{m} \mathbb{C}[u, \bar{u}]^{(n-j, n-j)}, \quad m \geq 1,
$$

and applying infinitesimal invariance. As we will see in the following sections, we are particularly interested in the cases $m=1,2$.

Remark 6.3. In (6.2) it is necessary to restrict consideration to $\bar{L}_{-m}$ for $m \geq 1$, because we actually need this derivative to fix $\rho_{\infty}$. Otherwise we cannot apply integration by parts to obtain integrals.

We will now give an example, where we compute the integrals for all elements of level 2. The single equation

$$
\bar{L}_{-2}\left(\rho_{0}^{2} u_{1}^{2}\right)=-6 u_{1} \bar{u}_{1}+14 u_{1}^{2} \bar{u}_{1}^{2}-8 u_{1}^{2} \bar{u}_{2}
$$

implies

$$
14 E\left(u_{1}^{2} \bar{u}_{1}^{2}\right)=6 E\left(u_{1} \bar{u}_{1}\right)+8 E\left(u_{1}^{2} \bar{u}_{2}\right)
$$

by infinitesimal invariance. Therefore,

$$
14 E\left(u_{1}^{2} \bar{u}_{1}^{2}\right)=3+8 E\left(u_{1}^{2} \bar{u}_{2}\right)
$$

by Theorem 1.7. On one hand,

$$
\bar{L}_{-1}\left(\rho_{0} u_{1}^{2} \bar{u}_{1}\right)=-3 u_{1}^{2} \bar{u}_{2}+2 u_{1} \bar{u}_{1} \text {. }
$$

On the other hand,

$$
L_{-1}\left(\rho_{0} \bar{u}_{1}^{2} u_{1}\right)=-3 \bar{u}_{1}^{2} u_{2}+2 u_{1} \bar{u}_{1} .
$$

Therefore,

$$
E\left(u_{1}^{2} \bar{u}_{2}\right)=E\left(u_{2} \bar{u}_{1}^{2}\right)=\frac{1}{3} .
$$

Equation (6.3) can now be used to obtain the following.

\section{Proposition 6.4.}

$$
E\left(u_{2} \bar{u}_{2}\right)=\frac{1}{3}, \quad E\left(u_{2} \bar{u}_{1}^{2}\right)=E\left(\bar{u}_{2} u_{1}^{2}\right)=\frac{1}{3}, \quad E\left(\left|u_{1}\right|^{4}\right)=\frac{17}{42} .
$$




\subsection{Expressions for $\bar{L}_{-1}$}

Consider (6.2) in the case $m=1$. The first expression we derive for this operator is purely algebraic.

Lemma 6.5. Suppose that $\sum k p_{k}=n$ and $\sum k q_{k}=n-1$ and let $u^{p} \bar{u}^{q}:=\prod_{k} u_{k}^{p_{k}} \bar{u}_{k}^{q_{k}}$. Then

$$
\begin{aligned}
\bar{L}_{-1}\left(\rho_{0} u^{p} \bar{u}^{q}\right)= & \left(\sum_{j \geq 1} j p_{j} u_{j}^{-1} u_{j-1} u^{p} \bar{u}^{q}\right)+\left(\left(2 \sum_{j \geq 1} q_{j}-2\right) \bar{u}_{1} u^{p} \bar{u}^{q}\right) \\
& -\left(\sum_{j \geq 1}(j+2) q_{j} \bar{u}_{j}^{-1} \bar{u}_{j+1} u^{p} \bar{u}^{q}\right) .
\end{aligned}
$$

The first sum of terms are of level $n-1$, and the other terms are of level $n$.

Proof. We calculate

$$
\begin{aligned}
\bar{L}_{-1}\left(\rho_{0} u^{p} \bar{u}^{q}\right)= & -\bar{u}_{1} u^{p} \bar{u}^{q}+\sum_{j \geq 1}\left(p_{j} u_{j}^{p_{j}-1} j\left(u_{j-1}-u_{1}^{*} u_{j}\right) \prod_{k \neq j} u_{k}^{p_{k}} \prod_{k \geq 1} \bar{u}_{k}^{q_{k}}\right. \\
& \left.+q_{j} \bar{u}_{j}^{q_{j}-1}(j+2)\left(u_{1} u_{j}-u_{j+1}\right)^{*} \prod_{k \geq 1} u_{k}^{p_{k}} \prod_{k \neq j} \bar{u}_{k}^{q_{k}}\right) \\
= & -\bar{u}_{1} u^{p} \bar{u}^{q}+\sum_{j \geq 1}\left(j p_{j} u_{j}^{-1} u_{j-1}+\left((j+2) q_{j}-j p_{j}\right) \bar{u}_{1}\right. \\
& \left.-(j+2) q_{j} \bar{u}_{j}^{-1} \bar{u}_{j+1}\right) \prod_{k} u_{k}^{p_{k}} \bar{u}_{k}^{q_{k}} .
\end{aligned}
$$

This simplifies to the expression in the statement of the lemma.

The second expression is in terms of divergence-type differential operators. We also note that the homogeneity condition on the domains can be expressed in terms of divergence-type operators.

Proposition 6.6. Let $n \geq 1$.

(a)

$$
\mathbb{C}[u]^{(n)}=\left\{P \in \mathbb{C}[u]: \sum_{j \geq 1} j u_{j} \frac{\partial P}{\partial u_{j}}=n P\right\} .
$$

(b) Suppressing $\rho_{0}$, the map

$$
\mathbb{C}[u]^{(n)} \otimes \mathbb{C}[\bar{u}]^{(n-1)} \stackrel{\operatorname{proj} \circ \bar{L}_{-1}}{\longrightarrow} \mathbb{C}[u]^{(n)} \otimes \mathbb{C}[\bar{u}]^{(n)}
$$

is of the form $1 \otimes \bar{R}_{1}$, where

$$
\bar{R}_{1}=\sum_{k \geq 1}\left(2 \bar{u}_{1} \bar{u}_{k}-(k+2) \bar{u}_{k+1}\right) \frac{\partial}{\partial \bar{u}_{k}}-2 \bar{u}_{1} .
$$

(b') The linear map $\bar{R}_{1}$ is injective. 
(c) Similarly,

$$
\mathbb{C}[u]^{(n)} \otimes \mathbb{C}[\bar{u}]^{(n-1)} \stackrel{\operatorname{proj} \circ \bar{L}_{-1}}{\longrightarrow} \mathbb{C}[u]^{(n-1)} \otimes \mathbb{C}[\bar{u}]^{(n-1)}
$$

is of the form $N_{1} \otimes 1$, where

$$
N_{1}=\sum_{j \geq 1} j u_{j-1} \frac{\partial}{\partial u_{j}} \text {. }
$$

Proof. We will prove $\left(\mathrm{b}^{\prime}\right)$ : If $n=1$, then $\bar{R}_{1}: \mathbb{C} \rightarrow \mathbb{C}[\bar{u}]^{(1)}$ is injective by dimension considerations. If $n \geq 2$, then consider the representation $\bar{\pi}$ of $\overline{\mathcal{W}}$ on $\mathbb{C}\left[\rho_{0}, u\right]$. For the lowest-weight representation generated by $\rho_{0}^{n-1}$, we have $c=0$ and $h=-\left(\frac{n-1}{2}\right)$ (see Section 4.6). This is a reducible Verma module if and only if

$$
-(n-1)=\frac{m^{2}-1}{12} .
$$

When the Verma module is irreducible, the creation operator $\bar{L}_{-1}$ is injective at each level, i.e. $\bar{R}_{1}$ is injective. Notice that the same thing would be true for $\bar{L}_{-k}$ for any $k>0$.

Remark 6.7. For $u^{P} \bar{u}^{Q} \in \mathbb{C}[u]^{(n)} \otimes \mathbb{C}[\bar{u}]^{(n)}$ such that $\bar{u}^{Q}$ is in the image of $\bar{R}_{1}$, there is a recursion formula

$$
E\left(u^{P} \bar{u}^{Q}\right)=E\left(\left(N_{1} \otimes 1\right)\left(u^{P} \bar{R}_{1}^{-1}\left(\bar{u}^{Q}\right)\right)\right)=E\left(N_{1}\left(u^{P}\right) \bar{R}_{1}^{-1}\left(\bar{u}^{Q}\right)\right)
$$

where we are denoting a partial inverse to $\bar{R}_{1}$ by $\bar{R}_{1}^{-1}$. Unfortunately this does not make any sense for most $Q$.

Definition 6.8. For a single complex variable $z$, we define $\frac{1}{\sqrt{k !}} z^{k}$ to be an orthonormal basis for $\mathbb{C}[z]$. For a tensor product such as

$$
\mathbb{C}[u]=\mathbb{C}\left[u_{1}\right] \otimes \mathbb{C}\left[u_{2}\right] \otimes \cdots
$$

we take the tensor product Hilbert space structure, meaning that $\frac{1}{\sqrt{p !}} u^{p}$ is an orthonormal basis, where

$$
p !:=p_{1} ! p_{2} ! \cdots .
$$

\section{Proposition 6.9.}

(a) The adjoint of

$$
\mathbb{C}[\bar{u}]^{(n-1)} \stackrel{\bar{R}_{1}}{\longrightarrow} \mathbb{C}[\bar{u}]^{(n)},
$$

where

$$
\bar{R}_{1}=\sum_{k \geq 1}\left(2 \bar{u}_{1} \bar{u}_{k}-(k+2) \bar{u}_{k+1}\right) \frac{\partial}{\partial \bar{u}_{k}}-2 \bar{u}_{1}
$$

is

$$
\mathbb{C}[\bar{u}]^{(n-1)} \stackrel{\bar{R}_{1}^{t}}{\longleftarrow} \mathbb{C}[\bar{u}]^{(n)}
$$

given by

$$
\bar{R}_{1}^{t}=\sum_{k \geq 1}\left(2 \bar{u}_{k} \frac{\partial}{\partial \bar{u}_{1}} \frac{\partial}{\partial \bar{u}_{k}}-(k+2) \bar{u}_{k} \frac{\partial}{\partial \bar{u}_{k+1}}\right)-2 \frac{\partial}{\partial \bar{u}_{1}} .
$$


(b) Let $K$ denote the kernel of $R_{1}^{t}$, i.e. the cokernel of $R_{1}$ (or the orthogonal complement of the image of $\left.R_{1}\right)$. Then

$$
0 \longleftarrow \mathbb{C}[u]^{(n-1)} \stackrel{R_{1}^{t}}{\longleftarrow} \mathbb{C}[u]^{(n)} \longleftarrow K \longleftarrow 0,
$$

i.e., $R_{1}^{t}$ is surjective.

(c)

$$
\begin{aligned}
\left(\operatorname{Image}\left(1 \otimes \bar{R}_{1}\right)+\operatorname{Image}\left(R_{1} \otimes 1\right)\right)^{\perp} & =\operatorname{kernel}\left(1 \otimes \bar{R}_{1}^{t}\right) \cap \operatorname{kernel}\left(R_{1}^{t} \otimes 1\right) \\
& =\mathbb{C}[u]^{(n)} \otimes \bar{K} \cap K \otimes \mathbb{C}[\bar{u}]^{(n)}=K \otimes \bar{K},
\end{aligned}
$$

which has dimension $(p(n)-p(n-1))^{2}>0$ for $n>1(p(\cdot)$ is the partition function).

Proof. Because of the normalization for the Hermitian inner product, the adjoint for multiplication by $z$ on $\mathbb{C}[z]$ is $\frac{\partial}{\partial z}$ on the $\mathbb{C}[z]$, and vice versa. This leads to the formula for $\bar{R}_{1}^{t}$.

Part (b) follows from the injectivity of $R_{1}$ (see ( $\left.\mathrm{b}^{\prime}\right)$ of Proposition 6.6).

Part (c) is elementary linear algebra: for the sum of two subspaces, the annihilators is the intersection of the annihilators.

Example 6.10. When $n=2$,

$\operatorname{kernel}\left(R_{1}^{t}\right)=\mathbb{C}\left\{u_{1}^{2}\right\}$.

When $n=3$,

kernel $\left(R_{1}^{t}\right)=\mathbb{C}\left\{u_{1}^{3}+2 u_{1} u_{2}\right\}$.

Note $p(3)-p(2)=3-2=1$.

When $n=4$,

kernel $\left(R_{1}^{t}\right)=\mathbb{C}\left\{4 u_{2}^{2}-6 u_{1} u_{3}, 3 u_{1}^{2}+16 u_{1}^{2} u_{2}+16 u_{1} u_{3}\right\}$.

Note $p(4)-p(3)=5-3=2$.

We will now give a slight generalization of Theorem 1.7 using the algebraic expression for $\bar{L}_{-1}$.

Corollary 6.11. Suppose that weight $(p)=n$. Then

$$
E\left(u^{p} \bar{u}_{n}\right)=\frac{1}{n+1} .
$$

Proof. The formula in Lemma 6.5 implies

$$
\bar{L}_{-1}\left(\rho_{0} u^{p} \bar{u}_{n-1}\right)=\sum_{j \geq 1} j p_{j} u_{j}^{-1} u_{j-1} u^{p} \bar{u}_{n-1}-(n+1) u^{p} \bar{u}_{n} .
$$

Thus we obtain a recursion relation

$$
(n+1) E\left(u^{p} \bar{u}_{n}\right)=\sum_{j \geq 1} j p_{j} E\left(u_{j}^{-1} u_{j-1} u^{p} \bar{u}_{n-1}\right) .
$$

The terms on the right hand side of the same form with weight $=n-1$. Since $\sum_{j \geq 1} j p_{j}=n$, induction implies the right hand side equals 1 . This implies the corollary. 


\subsection{Expressions for $\bar{L}_{-2}$}

We now consider the operator (6.2) in the case $m=2$, which is substantially more complicated than in the $m=1$ case. Recall that $p_{k}^{(-1)}$ denotes the Laurent coefficient of $\frac{z}{u(z)}$ and $P_{2}=$ $7 u_{1}^{2}-4 u_{2}$.

Proposition 6.12. Let $n \geq 2$.

(a) Suppressing $\rho_{0}^{2}$, the map

$$
\mathbb{C}[u]^{(n)} \otimes \mathbb{C}[\bar{u}]^{(n-2)} \stackrel{\operatorname{proj} \circ \bar{L}_{-2}}{\longrightarrow} \mathbb{C}[u]^{(n)} \otimes \mathbb{C}[\bar{u}]^{(n)}
$$

is of the form $1 \otimes \bar{R}_{2}$, where

$$
\bar{R}_{2}=2 \bar{P}_{2}-\sum_{j=1}^{\infty}\left(\bar{P}_{2} \bar{u}_{j}-3(j+2) \bar{u}_{1} \bar{u}_{j+1}+(j+3) \bar{u}_{j+2}-\bar{p}_{j+2}^{(-1)}\right) \frac{\partial}{\partial \bar{u}_{j}} .
$$

( $\left.a^{\prime}\right)$ The linear map $\bar{R}_{2}$ is injective.

(b) Similarly,

$$
\mathbb{C}[u]^{(n)} \otimes \mathbb{C}[\bar{u}]^{(n-2)} \stackrel{\text { proj } \circ \bar{L}_{-2}}{\longrightarrow} \bigoplus_{j=1}^{2} \mathbb{C}[u, \bar{u}]^{(n-j, n-j)}
$$

is of the form $N_{2} \otimes 1-3 N_{1} \otimes \bar{u}_{1}$, where

$$
N_{2}=\sum_{j \geq 2}(j-1) u_{j-2} \frac{\partial}{\partial u_{j}} \text {. }
$$

(c) If $u^{P} \otimes \bar{u}^{Q} \in \mathbb{C}[u, \bar{u}]^{(n, n)}$ such that $\bar{u}^{Q}$ lies in the image of $\bar{R}_{2}$, then

$$
E\left(u^{P} \bar{u}^{Q}\right)=E\left(N_{2}\left(u^{P}\right) \bar{R}_{2}^{-1}\left(\bar{u}^{Q}\right)\right)-3 E\left(N_{1}\left(u^{P}\right) \bar{u}_{1} \bar{R}_{2}^{-1}\left(\bar{u}^{Q}\right)\right) .
$$

Proof. The proof of $\left(a^{\prime}\right)$ is the same as $\left(b^{\prime}\right)$ of Proposition 6.6. Parts (a) and (b) follow by the formulas

$$
\bar{\pi}\left(L_{-2}\right)\left(\rho_{0}^{2}\right)=\bar{P}_{2}, \quad \rho_{0}^{2} \bar{\pi}\left(L_{-2}\right)\left(u_{j}\right)=\frac{j}{2} u_{j} \bar{P}_{2}-3 j u_{j-1} \bar{u}_{1}+(j-1) u_{j-2},
$$

and

$$
\rho_{0}^{2} \pi\left(L_{-2}\right)(u(z))=\frac{1}{u(z)}-\left(\frac{1}{z}-3 u_{1}\right) u^{\prime}(z)-\frac{1}{2} P_{2}\left(z u^{\prime}(z)+u(z)\right),
$$

which we then expand to obtain $\rho_{0}^{2} \pi\left(L_{-2}\right)\left(u_{j}\right)$.

Applying infinitesimal invariance to $\bar{L}_{-2}\left(\rho_{0}^{2} u^{P} \bar{u}^{Q}\right)$ gives part (c).

Proposition 6.13. Fix $n \geq 2$ and let $K_{m}=\operatorname{kernel}\left(R_{m}^{t}: \mathbb{C}[u]^{(n)} \rightarrow \mathbb{C}[u]^{(n-m)}\right)$ for $m=1,2$. Then

$$
K_{1} \cap K_{2}=\{0\}
$$

or

$$
\operatorname{image}\left(R_{1}\right)+\operatorname{image}\left(R_{2}\right)=\mathbb{C}[u]^{(n)} .
$$

Therefore, in principle, we can determine all moments by using only $\bar{L}_{-1}$ and $\bar{L}_{-2}$. 
Proof. Consider the cylic $\pi$-representation generated by $\rho_{0}^{n}$ :

$$
\pi(\mathcal{U}(\mathcal{W})) \rho_{0}^{n}=\bigoplus_{k=0}^{\infty} \rho_{0}^{n-k} \mathbb{C}[u]^{(k)}
$$

which is an irreducible Verma module. Therefore, the $n$-th graded component, $\mathbb{C}[u]^{(n)}$, has a basis consisting of elements of the form

$$
L_{-i_{j}} \cdots L_{-i_{1}}\left(\rho_{0}^{n}\right)
$$

where $0<i_{1} \leq \cdots \leq i_{j}$ and $i_{1}+\cdots+i_{j}=n$. The claim follows since $\mathcal{U}\left(\bigoplus_{k \geq 1} \mathbb{C} L_{-k}\right)$ is generated by $L_{-1}$ and $L_{-2}$.

Consider $u^{P} \otimes \bar{u}^{Q} \in \mathbb{C}[u]^{(n)} \otimes \mathbb{C}[\bar{u}]^{(n)}$. In principle, we can write

$$
\bar{u}^{Q}=\bar{R}_{1}\left(\bar{f}_{1}\right)+\bar{R}_{2}\left(\bar{f}_{2}\right)
$$

for some polynomials $\bar{f}_{j} \in \mathbb{C}[\bar{u}]^{(n-j)}$. We can then compute

$$
E\left(u^{P} \bar{u}^{Q}\right)=E\left(N_{1}\left(u^{P}\right)\left(\bar{f}_{1}-3 \bar{u}_{1} \bar{f}_{2}\right)\right)+E\left(N_{2}\left(u^{P}\right) \bar{f}_{2}\right) .
$$

The question now becomes how to divide $\bar{u}^{Q}$ into two pieces. In theory, this can be done using the orthogonal decomposition

$$
\mathbb{C}[\bar{u}]^{(n)}=\operatorname{image}\left(\bar{R}_{1}\right) \oplus\left(\operatorname{image}\left(\bar{R}_{2}\right) \ominus \operatorname{image}\left(\bar{R}_{1}\right)\right) .
$$

Remark 6.14. This gives a recursion relation for moments. The drawback is that we have to find all of the moments at a given level (indexed by $n$, which involves $u_{1}, \ldots, u_{n}$ ) to proceed to the next level. In implementing this procedure numerically (e.g. for the purpose of trying to reconstruct the distribution for $u_{1}$ ), we have found it convenient to not take the orthogonal complement, i.e. to work with an overdetermined system of linear equations. This has the advantage of providing consistency checks for all of our calculations. However, because $p(n)$ grows quite rapidly, this is slow (As of this writing, we do not have a conjecture for the distribution of $\left.u_{1}\right)$.

\subsection{Uniqueness of Werner's measures}

To close this section, we will now give an alternate proof of the uniqueness of Werner's family of measures (when $\nu_{0}$ is normalized to be a probability measure). Our statement is marginally stronger than Werner's, in that we only need to assume the measures are locally finite, i.e. $0<$ $\mu_{0}(\{a<|z|<A\})<\infty$ for some finite $0<a<A<\infty$ (this is implied by the nontriviality condition (1.1), but not vice versa).

Theorem 6.15. If there exists a family of locally finite measures $\left\{\mu_{S}\right\}$ on self-avoiding loops on Riemann surfaces which satisfies conformal restriction, then this family is unique up to multiplication by an overall positive constant.

Proof. We first claim that $\mu_{0}$ is uniquely determined (up to a constant which we can normalize). Conformal invariance of $\mu_{0}$ implies that there is a factorization as in Proposition 2.4:

$$
d \mu_{0}(\gamma)=d \nu_{0}(u) \times \frac{d \rho_{\infty}}{\rho_{\infty}},
$$

where here we view $d \nu_{0}$ as a measure on $u$. Local finiteness implies that $\nu_{0}$ is finite (see the proof of (b) of Proposition 2.1), and hence we can normalize it to be a probability measure. The 
measure $\nu_{0}$ is completely determined by the joint distributions of $u_{1}, \ldots, u_{N}, N \geq 1$. The $u_{j}$ are bounded, hence these distributions are determined by their joint moments. Finally we have shown, using only infinitesimal conformal invariance (which depends on the conformal restriction property, and not any specific features of Werner's construction), that the moments for these distributions are (in principle) computable. This determines $\mu_{0}$.

The proof that $\mu_{0}$ determines $\mu_{S}$ for all $S$ basically follows from the argument given in Section 6.1 of [17]. However there is a slight flaw in that argument. It is not quite the case that "The family of events of the type

$$
A_{D}=\{\gamma: \gamma \subset D \text { and goes around the hole in } D\},
$$

when $D$ varies in the family of annular regions in $S$ is stable under finite intersections". For example in the plane the intersection of the two annuli $A_{1}:=\Delta \backslash\{|z|<1 / 8\}$ and $A_{2}:=$ $\Delta \backslash\{|z-1 / 2|<1 / 8\}$ is a pair of pants; there does not exist an annulus inside of this pair of pants which contains all the loops which go around both holes. So the argument must be modified (this kind of argument is also used in the earlier proof of Lemma 4 of [17], and in that context it is valid, because 0 is always assumed to be in the hole of the allowed annuli).

Given knowledge of $\mu_{0}$, for any proper open subset $S$ of the plane, and for any nontrivial free homotopy class $C \subset \operatorname{Loop}(S), \mu_{S}(C)$ is uniquely determined; this follows from conformal restriction, because we can assume $S \subset \mathbb{C} \backslash\{0\}$ and all the loops in $C$ go around zero.

Suppose $S$ is a general Riemann surface. Consider the family of events $C=C_{D}$, where $C$ is a nontrivial free homotopy class of loops in an open subset $D \subset S$ such that $D$ is conformally equivalent to a proper open subset of $\mathbb{C}$. We claim this family is stable under finite intersections. Clearly $D_{1} \cap D_{2}$ is conformally equivalent to a proper open subset of $\mathbb{C}$. The main point is to show that $C_{1} \cap C_{2}$ determines a unique free homotopy class in $D_{1} \cap D_{2}$. This topological fact is probably well-known, but we will give a proof.

Suppose that we are given a fixed conformal equivalence of $D$ with a proper open subset of $\mathbb{C}, i: D \rightarrow \mathbb{C}$, and a free homotopy class $C \subset \operatorname{Loop}(D)$. If $\gamma \in \operatorname{Loop}(D)$, then (by the Jordan curve theorem applied to $i(\gamma))$ the complement of $i(D)$ is divided into an inside, $\operatorname{In}_{D}(\gamma)$, and an outside, $\operatorname{Out}_{D}(\gamma)$ (which contains $\infty$, i.e. large $z$ ).

Lemma 6.16. If $\gamma_{1} \in C_{D}$, then $C_{D}$ is determined by $\operatorname{In}_{D}\left(\gamma_{1}\right)$, i.e. if $\gamma_{2} \in \operatorname{Loop}(D)$, then $\gamma_{2} \in C_{D}$ if and only if $\operatorname{In}_{D}\left(\gamma_{2}\right)=\operatorname{In}_{D}\left(\gamma_{1}\right)$.

Proof. This is a topological claim, so in a standard way we can suppose loops are smooth, and intersections are transverse. Suppose $H(s, t)$ is a homotopy (with $\operatorname{Image}(H(\cdot, 0))=\gamma_{1}$, and Image $\left.(H(\cdot, 1))=\gamma_{2}\right)$. For $0<t<1, H(\cdot, t)$ is not necessarily simple, but we can nonetheless talk about $\operatorname{In}_{D}(H(\cdot, t))$, by using the inner boundary. This set, $\operatorname{In}_{D}(H(\cdot, t))$, is independent of $t$, by continuity, and this implies $\operatorname{In}_{D}\left(\gamma_{2}\right)=\operatorname{In}_{D}\left(\gamma_{1}\right)$.

Now consider the converse. Let $U_{ \pm}^{(j))}$ denote the bounded and unbounded components for $\mathbb{C} \backslash \gamma_{j}$, respectively. Then $U_{+}^{(1)} \cap U_{+}^{(2)}$ and $\{\infty\} \cup\left(U_{-}^{(1)} \cap U_{+}^{(2)}\right)$ are open contractible sets (for example $U_{+}^{(1)} \cap U_{+}^{(2)}$ is the bounded component for the inner boundary of $\gamma_{1} \cup \gamma_{2}$ ). The complement is a closed region with a boundary composed of the inner and outer boundaries for $\gamma_{1} \cup \gamma_{2}$, and it is homotopic to a annulus with boundary (for curves which intersect transversely, it is an annulus which is pinched at the points of intersection of the $\gamma_{i}$ ). This annular region is entirely contained in $D$, and hence $\gamma_{1}$ and $\gamma_{2}$ are homotopic in $D$.

We now use this to show that $C_{1} \cap C_{2}$ determines a unique free homotopy class in $D_{1} \cap D_{2}$. Fix conformal embeddings $i_{j}: D_{j} \rightarrow \mathbb{C}$, and use the restriction of $i_{1}$ to embed $D_{1} \cap D_{2}$. Suppose that $\gamma_{1}, \gamma_{2} \in C_{1} \cap C_{2}$. Then the Lemma implies that $\operatorname{In}_{D_{k}}\left(\gamma_{j}\right)$ does not depend on $j$. But then $\operatorname{In}_{D_{1} \cap D_{2}}\left(\gamma_{j}\right)$ is also independent of $j$, and hence by the Lemma, $\gamma_{1}$ and $\gamma_{2}$ are homotopic in $D_{1} \cap D_{2}$. 
This now implies that $C_{1} \cap C_{2}$ determines a unique free homotopy class in $D_{1} \cap D_{2}$. This class is clearly nontrivial, because its image in $D_{k}$ is nontrivial, $k=1,2$. This now implies that the set of events $C_{D}$ is stable under finite intersections. Now the argument in Section 6.1 of [17] implies $\mu_{S}$ is uniquely determined.

\section{The diagonal distribution}

To determine the joint distribution for $\left(\rho_{0}, \rho_{\infty}\right)$, Proposition 2.1 implies that it suffices to determine the distribution for $H=-\log (a) \geq 0$, which by part (e) of Proposition 2.1 is a kind of height function for

$$
\left\{\sigma \in \operatorname{Homeo}\left(S^{1}\right): \exists \text { unique welding } \sigma=l a u\right\} .
$$

Conjecture 7.1. For some $\beta_{0}<\frac{5 \pi^{2}}{4}$, the $\nu_{0}$ distribution for $a$ is given by

$$
\nu_{0}(\{\sigma: \exp (-x) \leq a(\sigma) \leq 1\})=\exp \left(-\beta_{0} / x\right), \quad x>0 .
$$

Equivalently the Laplace transform

$$
\int a^{\lambda} d \nu_{0}(\sigma)=\int_{x=0}^{\infty} a^{\lambda} d \exp \left(-\beta_{0} / x\right)=2 \sqrt{\lambda \beta_{0}} K_{1}\left(2 \sqrt{\lambda \beta_{0}}\right)
$$

for $\lambda>0$, where $K_{1}$ is a modified Bessel function.

We will first explain how this conjecture is related to a remarkable calculation of Werner in Section 7 of [17]. We will then present some calculations which are possibly relevant to a proof, and incidentally give an estimate for Werner's constant. Finally we will briefly indicate how the conjecture naturally generalizes to the deformation of Werner's measure considered in [13].

\subsection{A formula of Werner}

As in Section 7 of [17], consider the function

$$
F(\rho):=\mu\left(\operatorname{Loop}^{1}(A)\right),
$$

where $A$ is a finite type annulus with modulus $\rho=\rho(A)$, i.e. $\rho>0$ is the unique number such that $A$ is conformally equivalent to

$$
\left\{1<|z|<e^{\rho}\right\} .
$$

Cardy (see [6]) has conjectured an exact formula

$$
F(\rho)=6 \pi \frac{\sum_{k \in \mathbb{Z}}(-1)^{k-1} k q^{3 k^{2} / 2-k+1 / 8}}{\prod_{k=1}^{\infty}\left(1-q^{k}\right)}, \quad q=\exp \left(-2 \pi^{2} / \rho\right) .
$$

As we will explain below in more detail

$$
\operatorname{Loop}^{1}\left(\left\{1<|z|<e^{\rho}\right\}\right) \subset\left\{1 \leq \rho_{0} \leq \rho_{\infty} \leq e^{\rho}\right\} \subset \operatorname{Loop}^{1}\left(\left\{\frac{1}{4}<|z|<4 e^{\rho}\right\}\right)
$$

and as a consequence

$$
F(\rho) \leq \int_{0}^{\rho} \nu_{0}\left(e^{-x} \leq a \leq 1\right) d x \leq F(\log (16)+\rho) .
$$


This incidentally explains the constant $6 \pi$ in (7.2), which ensures that the derivative of $F$ is asymptotically one, or equivalently that $\nu_{0}$ is a probability measure.

Werner shows that $F(\rho)$ is asymptotic to const $\cdot \exp \left(-\frac{\beta}{\rho}\right)$ as $\rho \rightarrow 0$, where $\beta=\frac{5 \pi^{2}}{4}$; see Proposition 18 of [17]. This leads to the upper bound on $\beta_{0}$ in our statement of the diagonal distribution conjecture (if Cardy's conjecture is correct, then (7.4) implies sharper upper and lower bounds for $\beta_{0}$ ).

Lemma 7.2. Fix $x>0$. If $\gamma \in \operatorname{Loop}^{1}\left(\left\{1<|z|<e^{x}\right\}\right)$, then

$$
1 \leq \rho_{0}(\gamma) \leq \rho_{\infty}(\gamma) \leq e^{x}
$$

Proof. The Cauchy integral formula implies, for sufficiently smooth $\gamma$,

$$
\frac{1}{\rho_{0}}=\left(\phi_{+}^{-1}\right)^{\prime}(0)=\frac{1 !}{2 \pi i} \int_{\gamma} \frac{\phi_{+}^{-1}(t)}{t^{2}} d t
$$

Since $\gamma$ is outside the unit disk and $\phi_{+}^{-1}: U_{+} \rightarrow \Delta$. This implies

$$
\frac{1}{\rho_{0}} \leq \frac{\operatorname{length}(\gamma)}{2 \pi} \leq 1
$$

This implies the first inequality. The last inequality also follows from this.

We noted previously that the equality

$$
a^{2}=\frac{\rho_{0}^{2}}{\rho_{\infty}^{2}}=\frac{1-\sum_{m=1}^{\infty}(m-1)\left|b_{m}\right|^{2}}{1+\sum_{n=1}^{\infty}(n+1)\left|u_{n}\right|^{2}}
$$

implies $a \leq 1$, i.e. $\rho_{0} \leq \rho_{\infty}$.

\section{Lemma 7.3.}

(a)

$$
\mu_{0}\left\{\gamma: 1 \leq \rho_{0}(\gamma) \leq \rho_{\infty}(\gamma) \leq e^{x}\right\}=\int_{y=0}^{x} \nu_{0}\left\{e^{-y} \leq a \leq 1\right\} d y \leq x \nu_{0}\left\{e^{-x} \leq a \leq 1\right\}
$$

(b)

$$
\begin{aligned}
\int_{0}^{x} e^{-\beta_{0} / y} d y & =x e^{-\beta_{0} / x}-\Gamma\left(0, \frac{\beta_{0}}{x}\right)=x e^{-\beta_{0} / x}-E i\left(1, \frac{\beta_{0}}{x}\right) \\
& =x-\beta_{0} \log (x)+\beta_{0}\left(\log \left(\beta_{0}\right)+\gamma-1\right)-\frac{1}{2} \beta_{0}^{2} x^{-1}+\cdots
\end{aligned}
$$

(c) There is an asymptotic expansion

$$
\begin{aligned}
\int_{0}^{x} e^{-\beta_{0} / y} d y & =\frac{x^{2}}{\beta_{0}} e^{-\beta_{0} / x} \sum_{n=0}^{\infty}(-1)^{n} n !\left(\frac{x}{\beta_{0}}\right)^{n} \\
& =\frac{x^{2}}{\beta_{0}} e^{-\beta_{0} / x}\left(1-\frac{x}{\beta_{0}}+2 \frac{x^{2}}{\beta_{0}^{2}}-\cdots\right) \quad \text { as } \quad x \rightarrow 0 .
\end{aligned}
$$


Proof. (a) Using the factorization $d \mu_{0}=\frac{d \rho_{\infty}}{\rho_{\infty}} \times d \nu_{0}$,

$$
\begin{aligned}
\mu_{0}\left\{\gamma: 1 \leq \rho_{0}(\gamma) \leq \rho_{\infty}(\gamma) \leq e^{x}\right\} & =\mu_{0}\left\{\frac{1}{\rho_{\infty}} \leq a \leq 1,1 \leq \rho_{\infty} \leq e^{x}\right\} \\
& =\int_{\rho_{\infty}=1}^{e^{x}} \nu_{0}\left\{\frac{1}{\rho_{\infty}} \leq a \leq 1\right\} \frac{d \rho_{\infty}}{\rho_{\infty}} .
\end{aligned}
$$

By making the change of variables $\rho_{\infty}=e^{y}$, we obtain the expression in part (a).

(b) and (c) are standard facts. For example there is a Laurent expansion

$$
e^{-\beta_{0} / x}=1-\frac{\beta_{0}}{x}+\frac{1}{2}\left(\frac{\beta_{0}}{x}\right)^{2}-\cdots, \quad 0<|x|<\infty .
$$

Therefore there is an expansion

$$
\begin{aligned}
\int_{0}^{x} e^{-\beta_{0} / y} d y= & x-\beta_{0} \log (x)+c_{0}-\frac{1}{2} \beta_{0}^{2} x^{-1}+\frac{\beta_{0}^{3}}{3 ! 2} x^{-2}-\cdots \\
& -(-1)^{n} \frac{1}{n !(n-1)} \beta_{0}^{n} \frac{1}{x^{n-1}}-\cdots
\end{aligned}
$$

where the divergence of the logarithm and the Laurent expansion at $x=0$ perfectly cancel, allowing us to figure out $c_{0}$.

\section{Corollary 7.4.}

$$
\mu_{0}\left(\operatorname{Loop}^{1}\left(\left\{1<|z|<e^{\rho}\right\}\right)\right) \leq \rho \nu_{0}\left\{e^{-\rho} \leq a \leq 1\right\} .
$$

Proof. This follows from Lemma 7.2 and (a) of Lemma 7.3.

Here is another approach, although not quite as sharp:

$$
\operatorname{Loop}^{1}\left(\left\{1<|z|<e^{\rho}\right\}\right) \subset \operatorname{Loop}^{1}\left(\left\{|z|<e^{\rho}\right\}\right) \backslash \operatorname{Loop}(\Delta) .
$$

Werner's formula for the measure of the latter set is $c_{\mathrm{W}} \rho$, where $c_{\mathrm{W}}$ is Werner's constant (see below).

\subsection{Werner's constant}

Recall that we have normalized Werner's family of measures by assuming that $\nu_{0}$ is a probability measure. We let $c_{\mathrm{W}}$ denote the constant such that if $\gamma$ is a loop which surrounds $\Delta$,

$$
\mu_{0}\left(\operatorname{Loop}^{1}\left(U_{+} \backslash\{0\}\right) \backslash \operatorname{Loop}(\Delta)\right)=c_{\mathrm{W}} \log \left(\rho_{0}(\gamma)\right) .
$$

Proposition 7.5. $c_{\mathrm{W}} \geq 1$.

Proof. On the one hand

$$
\operatorname{Loop}^{1}\left(\left\{1<|z|<e^{\rho}\right\}\right) \subset \operatorname{Loop}^{1}\left(\left\{|z|<e^{\rho}\right\}\right) \backslash \operatorname{Loop}(\Delta) .
$$

Therefore by Werner's formula for the measure of the latter set,

$$
F(\rho) \leq c_{\mathrm{W}} \rho .
$$

On the other hand

$$
\operatorname{Loop}^{1}\left(\left\{1<|z|<e^{\rho}\right\}\right) \subset\left\{1 \leq \rho_{0} \leq \rho_{\infty} \leq e^{\rho}\right\} \subset \operatorname{Loop}^{1}\left(\left\{\frac{1}{4}<|z|<4 e^{\rho}\right\}\right),
$$


where the last inclusion uses Koebe's quarter theorem. Therefore

$$
F(\rho) \leq \int_{0}^{\rho} \nu_{0}\left(e^{-x} \leq a \leq 1\right) d x \leq F(\log (16)+\rho) .
$$

Because

$$
\nu_{0}\left(e^{-x} \leq a \leq 1\right) \uparrow 1 \quad \text { as } \quad x \uparrow 1
$$

it follows that $F(\rho)$ behaves like a linear function with slope one for $\rho \gg 1$. This behavior is compatible with the estimate above using Werner's formula if and only if $c_{\mathrm{W}} \geq 1$. This implies the proposition.

\subsection{Some ideas}

The conjectural Laplace transform (7.1) satisfies the ODE

$$
\lambda f^{\prime \prime}(\lambda)-\beta_{0} f(\lambda)=0
$$

Thus we need to show that

$$
\int\left(\lambda \log (a)^{2}-\beta_{0}\right) a^{\lambda} d \nu_{0}(\sigma)=0, \quad \lambda>0
$$

for some constant $\beta_{0}$. Roughly speaking, we are trying to calculate the second moment for the distribution of $H=-\log (a)$. To calculate the second moment for a standard normal complex variable, one can apply $\partial \bar{\partial}$ to $\exp \left(-|z|^{2} / 2\right)$ and use infinitesimal invariance of the background Lebesgue measure; our strategy is to do the same with the stress tensor $T(t)$ in place of $\partial, a^{\lambda}$ in place of the Gaussian, and Werner's measure in place of Lebesgue measure.

We will now list a number of formulas which are hopefully useful.

\section{Lemma 7.6.}

(a) For $n>0$

$$
L_{n} L_{-n} a^{\lambda}=L_{-n} L_{n} a^{\lambda}=\frac{\lambda^{2}}{4} P_{n}\left(l_{1}, \ldots, l_{n}\right) P_{n}\left(u_{1}, \ldots, u_{n}\right) a^{\lambda-n}-n \lambda a^{\lambda} .
$$

(b) For $m>n \geq 0$

$$
L_{m} L_{-n} a^{\lambda}=\frac{\lambda^{2}}{4} P_{m}\left(l_{1}, \ldots, l_{m}\right) P_{n}\left(u_{1}, \ldots, u_{n}\right) a^{\lambda} \frac{\rho_{\infty}^{m}}{\rho_{0}^{n}} .
$$

(c)

$$
\begin{aligned}
\vec{L}_{n} \vec{L}_{-n} a^{\lambda} & =\vec{L}_{-n} \vec{L}_{n} a^{\lambda}=\lambda^{2} \operatorname{Re}\left(P_{n}\left(l_{1}, \ldots, l_{n}\right)\right) \operatorname{Re}\left(P_{n}\left(u_{1}, \ldots, u_{n}\right)\right) a^{\lambda-n}-2 n \lambda a^{\lambda} \\
& =\lambda^{2} \operatorname{Re}\left(P_{n}\left(u_{1}\left(\sigma^{-1}\right), \ldots, u_{n}\left(\sigma^{-1}\right)\right)\right) \operatorname{Re}\left(P_{n}\left(u_{1}, \ldots, u_{n}\right)\right) a^{\lambda-n}-2 n \lambda a^{\lambda}
\end{aligned}
$$

(d) For $m>n \geq 0$

$$
\vec{L}_{m} \vec{L}_{-n} a^{\lambda}=\lambda^{2} \operatorname{Re}\left(P_{m}\left(l_{1}, \ldots, l_{m}\right)\right) \operatorname{Re}\left(P_{n}\left(u_{1}, \ldots, u_{n}\right)\right) a^{\lambda} \frac{\rho_{\infty}^{m}}{\rho_{0}^{n}} .
$$


Proof. (a) The fact that $L_{n}$ and $L_{-n}$ commute when acting on $a^{\lambda}$ follows from the fact that $L_{0} a=0$.

Using $L_{-n}\left(\rho_{\infty}\right)=0$ and (d) of Proposition 4.1,

$$
\left.L_{-n} a^{\lambda}=\lambda a^{\lambda-1} L_{-n}\left(\rho_{0}\right) \frac{1}{\rho_{\infty}}=\frac{\lambda}{2 \rho_{0}^{n}} P_{n}(u)\right) a^{\lambda},
$$

where we have abbreviated $P_{n}\left(u_{1}, \ldots, u_{n}\right)=P_{n}(u)$. Therefore

$$
L_{n} L_{-n} a^{\lambda}=\frac{\lambda}{2 \rho_{0}^{n}}\left(\lambda a^{\lambda-1} \rho_{0} L_{n}\left(\frac{1}{\rho_{\infty}}\right) P_{n}\left(u_{1}, \ldots, u_{n}\right)+a^{\lambda} L_{n}\left(P_{n}\left(u_{1}, \ldots, u_{n}\right)\right)\right) .
$$

Recall that $P_{n}\left(u_{1}, \ldots, u_{n}\right)=-2 n u_{n}+$ function $\left(u_{1}, \ldots, u_{n-1}\right)$ and $L_{n}\left(u_{n}\right)=\rho_{0}^{n}$. This implies

$$
L_{n} L_{-n} a^{\lambda}=\frac{\lambda}{2 \rho_{0}^{n}}\left(\lambda a^{\lambda-1} \rho_{0} \frac{1}{2}\left(\frac{1}{\rho_{\infty}}\right)^{-n+1} P_{n}(l) P_{n}(u)+a^{\lambda}\left(-2 n \rho_{0}^{n}\right)\right)
$$

This simplifies to (a).

(b) This follows in a similar way, using the fact that $L_{m}$ kills $P_{n}\left(u_{1}, \ldots, u_{n}\right)$.

(c) and (d) are proven in a similar way, and will not be used.

Recall that

$$
\left(\partial \log \left(\phi_{+}^{-1}\right)\right)^{2}=\left(\sum_{n=0}^{\infty} P_{n}\left(\phi_{+}\right) t^{n}\right)\left(\frac{d t}{t}\right)^{2}
$$

(this is a holomorphic quadratic differential which is well-defined in $U_{+}$) and

$$
\left(\partial \log \left(\phi_{-}^{-1}\right)\right)^{2}=\left(\sum_{n=0}^{\infty} P_{n}\left(\phi_{-}\right) t^{-n}\right)\left(\frac{d t}{t}\right)^{2}
$$

(this is a holomorphic quadratic differential which is well-defined in $U_{-}$; note that

$$
\left(\frac{d t}{t}\right)^{2}=\left(\frac{d t^{-1}}{t^{-1}}\right)^{2}
$$

The fact that these two quadratic differentials do not have a common domain, or at the very best, are possibly defined on the rough loop $\gamma$, is a crucial point.

\section{Proposition 7.7.}

$$
E\left(\left(\lambda P_{n}\left(\phi_{+}\right) P_{n}\left(\phi_{-}\right)-4 n\right) a^{\lambda}\right)=E\left(\left(\lambda P_{n}(u) P_{n}(l) a^{-n}-4 n\right) a^{\lambda}\right)=0 .
$$

Proof. This follows from the Lemma and infinitesimal conformal invariance.

The basic question now is whether there is a constant $\beta_{0}$ such that $\lambda \log (a)^{2}-\beta_{0}$ is a limit, in an appropriate measure theoretic sense relative to $\nu_{0}$, of linear combinations of the functions $\lambda P_{n}\left(\phi_{+}\right) P_{n}\left(\phi_{-}\right)-2 n$, as $n$ varies.

Question 7.8. Do there exist constants $c_{n}$ such that

$$
\sum_{n=1}^{N} c_{n} P_{n}(u) P_{n}(l) a^{-n} \rightarrow \log (a)^{2} \quad \text { as } \quad N \rightarrow \infty
$$

in some measure-theoretic sense relative to $\nu_{0}$ ? 
This is definitely false for all $\sigma$. To see this, suppose that

$$
\sigma=\phi_{N}\left(w_{N}, z\right)=z \frac{\left(1+\overline{w_{N}} z^{-N}\right)^{1 / N}}{\left(1+w_{N} z^{N}\right)^{1 / N}} .
$$

In this case

$$
u(z)=z\left(1+w_{N} z^{N}\right)^{-1 / N}, \quad \frac{U^{\prime}(t)}{U(t)}=\frac{1}{t} \frac{1}{1-w_{N} t^{N}}
$$

and

$$
(\partial \log U(t))^{2}=\left(1+2 w_{N} t^{N}+3\left(w_{N} t^{N}\right)^{2}+4\left(w_{N} t^{N}\right)^{3}+\cdots\right)\left(\frac{d t}{t}\right)^{2} .
$$

Thus for this particular $u$

$$
P_{n}(u)=(m+1) w_{N}^{m}=-P_{n}(l)^{*}, \quad n=m N
$$

and zero otherwise. Also

$$
\begin{aligned}
& l(t)=t\left(1+\bar{w}_{N} t^{-N}\right), \quad \partial \log (l(t))=\frac{1}{t\left(1+\bar{w}_{N} t^{-N}\right)} d t=\frac{t^{N-1}}{t^{N}+\bar{w}_{N}} d t, \\
& a=\left(1-\left|w_{N}\right|^{2}\right)^{1 / N},
\end{aligned}
$$

so that

$$
\log (a)^{2}=\frac{1}{N^{2}} \log \left(1-\left|w_{N}\right|^{2}\right)^{2} .
$$

If we actually have an identity, then for each $N=1,2, \ldots$

$$
\frac{1}{N^{2}} \log \left(1-\left|w_{N}\right|^{2}\right)^{2}=\sum_{m=0}^{\infty} c_{m N} \frac{(m+1)^{2}\left|w_{N}\right|^{2 m}}{\left(1-\left|w_{N}\right|^{2}\right)^{m}} .
$$

If we set $x=\left|w_{N}\right|^{2}$, then this is equivalent to

$$
\log (1-x)^{2}=\sum_{m=0}^{\infty} N^{2} c_{m N}(m+1)^{2}\left(\frac{x}{1-x}\right)^{m} .
$$

This is clearly impossible: we cannot consistently solve for the constants. Furthermore the radius of convergence for the 1.h.s. is 1 , and the radius of convergence for the r.h.s. is $\frac{1}{2}$.

A more promising approach seems to be to use the stress-energy tensor. Here is one heuristic calculation:

$$
\begin{aligned}
E(T(t) & \left.T(s) a^{\lambda}\right)=\sum_{n, m} E\left(L_{-n} L_{-m} a^{\lambda}\right) t^{n} s^{m}\left(\frac{d t}{t}\right)^{2}\left(\frac{d s}{s}\right)^{2} \\
= & \sum_{n} E\left(L_{-n} L_{n} a^{\lambda}\right) t^{n} s^{-n}\left(\frac{d t}{t}\right)^{2}\left(\frac{d s}{s}\right)^{2} \\
& \times \lambda \sum_{n} E\left(\left(\lambda P_{n}\left(\phi_{+}\right) P_{n}\left(\phi_{-}\right)-n\right) a^{\lambda}\right) t^{n} s^{-n}\left(\frac{d t}{t}\right)^{2}\left(\frac{d s}{s}\right)^{2} \\
= & \lambda\left(\lambda E\left(\left(\partial \log \left(\phi_{+}^{-1}(t)\right)\right)^{2}\left(\partial \log \left(\phi_{+}^{-1}(s)\right)\right)^{2} a^{\lambda}\right)-\sum_{n} n\left(\frac{t}{s}\right)^{n}\left(\frac{d t}{t}\right)^{2}\left(\frac{d s}{s}\right)^{2} E\left(a^{\lambda}\right)\right) \\
= & \lambda\left(\lambda E\left(\left(\partial \log \left(\phi_{+}^{-1}(t)\right)\right)^{2}\left(\partial \log \left(\phi_{+}^{-1}(s)\right)\right)^{2} a^{\lambda}\right)-\delta^{\prime}\left(\frac{t}{s}\right)\left(\frac{d t}{t}\right)^{2}\left(\frac{d s}{s}\right)^{2} E\left(a^{\lambda}\right)\right) .
\end{aligned}
$$

We now need to apply some kind of pairing for quadratic differentials, and we are stymied at this point. 


\subsection{KS conjecture and diagonal distribution}

In [13] Kontsevich and Suhov show that for each Riemann surface, there exists a continuous positive determinant line bundle Det $\rightarrow \operatorname{Loop}(S)$, and these line bundles have a natural restriction property. They conjecture that for each "central charge" $c$ (in some range), there exists a family of measures $\mu_{S}$ having values in the positive line bundle Det $^{c}$ and satisfying a conformal restriction property. In the case $c=0$, this family is the family of measures constructed by Werner.

There is a canonical trivialization of the determinant line bundle in genus zero, so that the conjectured KS measure can be viewed as a scalar measure which is invariant with respect to global conformal transformations; see Section 2.5 of [13]. We denote this measure restricted to $\operatorname{Loop}^{1}(\mathbb{C} \backslash\{0\})$ by $\mu_{c}$; properly normalized, this is the Werner measure when $c=0$.

Lemma 7.9. Assume that $\mu_{c}$ exists. Then

(a) The distributions for $\rho_{0}$ and $\rho_{\infty}$ are scale invariant.

(b)

$$
d\left(W_{*} \mu\right)\left(\sigma, \rho_{\infty}\right)=d \nu_{c}(\sigma) \times \frac{d \rho_{\infty}}{\rho_{\infty}} .
$$

(c) The measure $d \nu_{c}(\sigma)$ is inversion invariant and invariant with respect to conjugation by $C: z \mapsto z^{*}$.

(d) The measure $d \nu_{c}(\sigma)$ is supported on $\sigma$ having triangular factorization $\sigma=$ lau, i.e. $m=1$.

(e) If in addition $\nu_{c}$ is finite, and hence can be normalized to be a probability measure, then there is an inequality generalizing (7.4),

$$
F_{c}(\rho) \leq \int_{0}^{\rho} \nu_{c}\left(e^{-x} \leq a \leq 1\right) d x \leq F_{c}(\log (16)+\rho),
$$

where $F_{c}(\rho):=\mu_{c}\left(\operatorname{Loop}^{1}(A)\right)$, where $A$ is a finite type annulus with modulus $\rho=\rho(A)$.

This is a rigorous result (contingent on the existence of $\mu_{c}$ ), because (a)-(d) use only global conformal invariance of $\mu_{c}$, and (e) only depends on (7.3).

There is a natural conjecture for the diagonal distribution (there may be a conjecture for $F_{c}(\rho)$ which is implicit in [6], but we will not pursue this).

Conjecture 7.10. The $\nu_{c}$ distribution for $H=-\log (a)$ is the inverse gamma distribution with parameters $\alpha=1-c$ and some $\beta_{c}>0$ (possibly proportional to $h^{+}(c)$, the larger value of two values of the conformal anomaly $h$ corresponding to $c<1$ ). In other words we are conjecturing that

$$
\nu_{c}(\{\sigma: \exp (-x) \leq a(\sigma) \leq 1\})=\frac{\Gamma\left(\alpha, \beta_{c} / x\right)}{\Gamma(\alpha)}, \quad x>0
$$

and the Laplace transform

$$
\int a^{\lambda} d \nu_{c}(\sigma)=\frac{2\left(\beta_{c} \lambda\right)^{\frac{\alpha}{2}}}{\Gamma(\alpha)} K_{\alpha}\left(\sqrt{4 \beta_{c} \lambda}\right)
$$

where $K_{\alpha}$ is a modified Bessel function. This function of $\lambda$ satisfies the differential equation

$$
\lambda f^{\prime \prime}(\lambda)+c f^{\prime}(\lambda)-\beta_{c} f(\lambda)=0 .
$$


This differential equation obviously makes sense for values of the parameters which are not necessarily positive. But for example if $c=1$, i.e. $\alpha=0$, then the particular solution we are considering, $K_{0}$, is not finite at $\lambda=0$, so that the probabilistic interpretation is lost (this is obvious by noting that the pdf is not integrable at $\infty$ when $\alpha=0)$. In terms of our conjecture this means that when $c=1$, the $\sigma$ distribution for the conjectured Kontsevich-Suhov measure is not finite, according to us.

To motivate this, in a heuristic way, we imagine that $\mu_{c}$ is absolutely continuous with respect to Werner's measure $\mu_{0}: \mu_{c}=\delta_{c} d \mu_{0}$. We then apply infinitesimal invariance in the following way. Suppose that $n>0$. Then

$$
\begin{gathered}
L_{n}\left(L_{-n}\left(a^{\lambda}\right) \delta^{c} \delta\left(\rho_{0}=1\right) d \mu_{0}\right)=\left(\left(L_{n} L_{-n}\left(a^{\lambda}\right)\right) \delta^{c}+L_{-n}\left(a^{\lambda}\right) L_{n}\left(\delta^{c}\right)\right) \delta\left(\rho_{0}=1\right) d \mu_{0} \\
=\left(\left(\lambda^{2} P_{n}(u) P_{n}(l) a^{-n}-2 n \lambda\right)+\lambda P_{n}(l) \rho_{\infty}^{n} c Q_{n}(u, l)\right) \delta\left(\rho_{0}=1\right) a^{\lambda} \delta^{c} d \mu_{c},
\end{gathered}
$$

where we have tentatively written

$$
L_{n}\left(\delta_{c}\right)=c Q_{n}(u, l) \delta_{c} .
$$

This should rigorously be expressed in terms of divergences, as proposed in Section 2.5.2 of [13]. From this, by dividing by $\lambda$, we can deduce that

$$
\int\left(\lambda P_{n}(u) P_{n}(l) a^{-n}+c P_{n}(l) Q_{n}(u, l) \rho_{\infty}^{n}-2 n\right) a^{\lambda} d \nu_{c}=0 .
$$

Now we would have to take linear combinations and limits, to obtain $\log (a)^{2}$ from the first term, $\log (a)$ from the second term (involving $c$ ), and a constant $\beta_{c}$ from the third term.

\section{A The Vietoris topology}

Suppose that $S$ is a topological space. The Vietoris topology on $\operatorname{Comp}(S)$ has a base consisting of sets of the form

$$
\left\{K \in \operatorname{Comp}(S): K \subset U, K \cap U_{i} \neq \phi, i=1, \ldots, n\right\},
$$

where $U, U_{1}, \ldots, U_{n}$ are open subsets of $S$. Given $K_{0} \in \operatorname{Comp}(S)$, suppose we tightly cover $K_{0}$ with open sets $U_{i}, 1 \leq i \leq n$, and let $U=\cup_{i} U_{i}$. Then " $K$ is close to $K_{0}$ " means that (i) $K \subset U$, so every point in $K$ is close to a point in $K_{0}$, and (ii) for each point $x_{0} \in K_{0}, x_{0} \in U_{i}$, for some $i$, hence $K \cap U_{i} \neq \varnothing$ implies $x_{0}$ is close to some point in $K$. If $S$ is metrizable, with metric $d$, then the Vietoris topology is compatible with the associated Hausdorff metric topology on $\operatorname{Comp}(S)$, where the Hausdorff metric is given by

$$
\delta\left(K_{1}, K_{2}\right)=\max \left\{\sup _{p_{1} \in K_{1}}\left(d\left(p_{1}, K_{2}\right)\right), \sup _{p_{2} \in K_{2}}\left(d\left(K_{1}, p_{2}\right)\right)\right\} .
$$

For most topological properties $\tau, " S$ is $\tau$ " if and only if "Comp $(S)$ is $\tau$ " (see Section 4 of [14]). In particular if $S$ is second countable and locally compact, then $\operatorname{Comp}(S)$ is second countable and locally compact.

Suppose that $S$ is a Riemann surface with a fixed compatible complete metric. The associated Hausdorff metric on $\operatorname{Loop}(S)$ is obviously not complete, since for example a small circle can pinch down to a point. Does there exist a complete separable metric on $\operatorname{Loop}(S)$ compatible with the Vietoris topology?

\section{Acknowledgements}

We thank Tom Kennedy for useful conversations, and the referees for many useful suggestions regarding exposition and inclusion of references. 


\section{References}

[1] Airault H., Malliavin P., Thalmaier A., Brownian measures on Jordan-Virasoro curves associated to the Weil-Petersson metric, J. Funct. Anal. 259 (2010), 3037-3079.

[2] Astala K., Jones P., Kupiainen A., Saksman E., Random curves by conformal welding, C. R. Math. Acad. Sci. Paris 348 (2010), 257-262, arXiv:0912.3423.

[3] Bauer R.O., A simple construction of Werner measure from chordal SLE $\mathrm{S}_{8 / 3}$, Illinois J. Math. 54 (2010), 1429-1449, arXiv:0902.1626.

[4] Benoist S., Dubédat J., An SLE 2 loop measure, arXiv:1405.7880.

[5] Bishop C.J., Conformal welding and Koebe's theorem, Ann. of Math. 166 (2007), 613-656.

[6] Cardy J., The O(n) model on the annulus, J. Stat. Phys. 125 (2006), 1-21, math-ph/0604043.

[7] Di Francesco P., Mathieu P., Sénéchal D., Conformal field theory, Graduate Texts in Contemporary Physics, Springer-Verlag, New York, 1997.

[8] Duren P.L., Univalent functions, Grundlehren der Mathematischen Wissenschaften, Vol. 259, SpringerVerlag, New York, 1983.

[9] Duren P.L., Schiffer M., The theory of the second variation in extremum problems for univalent functions, J. Analyse Math. 10 (1962/1963), 193-252.

[10] Hille E., Analytic function theory. Vol. II, Introductions to Higher Mathematics, Ginn and Co., Boston, Mass. - New York - Toronto, Ont., 1962.

[11] Kac V.G., Raina A.K., Bombay lectures on highest weight representations of infinite-dimensional Lie algebras, Advanced Series in Mathematical Physics, Vol. 2, World Scientific Publishing Co., Inc., Teaneck, NJ, 1987.

[12] Kirillov A.A., Yuriev D.V., Representations of the Virasoro algebra by the orbit method, J. Geom. Phys. 5 (1988), 351-363.

[13] Kontsevich M., Suhov Y., On Malliavin measures, SLE, and CFT, Proc. Steklov Inst. Math. 258 (2007), 100-146, math-ph/0609056.

[14] Michael E., Topologies on spaces of subsets, Trans. Amer. Math. Soc. 71 (1951), 152-182.

[15] Segal G., The definition of conformal field theory, in Topology, Geometry and Quantum Field Theory, Proceedings of the Symposium in Honour of the 60th Birthday of Graeme Segal (Oxford, June 24-29, 2002), London Mathematical Society Lecture Note Series, Vol. 308, Editor U. Tillmann, Cambridge University Press, Cambridge, 2004, 421-577.

[16] Simon B., OPUC on one foot, Bull. Amer. Math. Soc. (N.S.) 42 (2005), 431-460, math.SP/0502485.

[17] Werner W., The conformally invariant measure on self-avoiding loops, J. Amer. Math. Soc. 21 (2008), 137-169, math.PR/0511605. 